\title{
Decays of a NMSSM CP-odd Higgs in the low-mass region
}

\author{
Florian Domingo \\ Instituto de Física Teórica (UAM/CSIC), Universidad Autónoma de Madrid, \\ Cantoblanco, E-28049 Madrid, Spain \\ Instituto de Física de Cantabria (CSIC-UC), \\ E-39005 Santander, Spain \\ E-mail: florian.domingo@desy.de
}

AbSTRACT: A popular regime in the NMSSM parameter space involves a light CP-odd Higgs $A_{1}$. This scenario has consequences for e.g. light singlino Dark Matter annihilating in the $A_{1}$-funnel. In order to confront the pseudoscalar to experimental limits such as flavour observables, Upsilon decays or Beam-Dump experiments, it is necessary to control the interactions of this particle with hadronic matter and derive the corresponding decays. The partonic description cannot be relied upon for masses close to $m_{A_{1}} \sim 1 \mathrm{GeV}$ and we employ a chiral lagrangian, then extended to a spectator model for somewhat larger masses, to describe the interplay of the CP-odd Higgs with hadrons. Interestingly, a mixing can develop between $A_{1}$ and neutral pseudoscalar mesons, leading to substantial hadronic decays and a coupling of $A_{1}$ to the chiral anomaly. Additionally, quartic $A_{1}$-meson couplings induce tri-meson decays of the Higgs pseudoscalar. We investigate these effects and propose an estimate of the Higgs widths for masses below $m_{A_{1}} \lesssim 3 \mathrm{GeV}$. While we focus on the case of the NMSSM, our results are applicable to a large class of models.

Keywords: Supersymmetry Phenomenology

ARXIV EPRINT: 1612.06538 


\section{Contents}

1 The NMSSM and a light CP-odd Higgs 1

2 From the partonic lagrangian to the mesonic interactions 4

2.1 Partonic lagrangian below the $c \bar{c}$ threshold 4

2.2 Chiral lagrangian 5

2.3 Low-energy coupling constants — meson masses 7

3 Decays of a CP-odd Higgs from the chiral lagrangian 8

$3.1 A_{1}$-meson mixing 8

$\begin{array}{lll}3.2 & \text { Photonic decay } & 10\end{array}$

$\begin{array}{lll}3.3 & \text { Leptonic decays } & 11\end{array}$

$\begin{array}{lll}3.4 & \text { Tri-meson decays } & 13\end{array}$

$\begin{array}{ll}3.5 & \text { Radiative hadronic decays } \\ 3.6\end{array}$

$\begin{array}{ll}3.6 & \text { Decays in the chiral limit: summary }\end{array}$

4 Beyond the chiral limit $\quad 18$

\section{The NMSSM and a light CP-odd Higgs}

While the hunt for physics beyond the Standard Model at the high-energy frontier continues at the LHC or in Dark Matter experiments - with disappointing results so far —, new physics may still have a few surprises in store in the low-mass region. Axion-phenomenology is a classical example of such effects in the limit of light less-than-weakly-coupled particles. In the following, we consider another case of comparatively light state occurring in the context of the Next-to-Minimal Supersymmetric Standard Model (NMSSM) [1], a wellmotivated extension of the Standard Model (SM).

In the NMSSM, the CP-odd Higgs sector (ignoring the Goldstone boson) consists of two degrees of freedom - a doublet component $A^{0}$, comparable to the MSSM pseudoscalar, and a singlet state $A_{S}^{0}$. Both mix at tree-level according to the following mass-matrix:

$$
\begin{aligned}
& \mathcal{M}_{\text {CP-odd }}^{2}=\left(\begin{array}{cc}
\frac{2 \lambda s}{\sin 2 \beta}\left(A_{\lambda}+\kappa s\right) & \lambda v\left(A_{\lambda}-2 \kappa s\right) \\
\lambda v\left(A_{\lambda}-2 \kappa s\right) & -3 \kappa s A_{\kappa}+\frac{\lambda v^{2} \sin 2 \beta}{2 s}\left(A_{\lambda}+4 \kappa s\right)
\end{array}\right) \\
& =P \cdot \operatorname{diag}\left(m_{A_{1}}^{2}, m_{A_{2}}^{2}\right) \cdot P^{T} \quad P \equiv\left(\begin{array}{cc}
\cos \theta_{P} & -\sin \theta_{P} \\
\sin \theta_{P} & \cos \theta_{P}
\end{array}\right)
\end{aligned}
$$

where $\lambda$ and $\kappa$ are parameters from the superpotential, $A_{\lambda}$ and $A_{\kappa}$, parameters from the soft supersymmetry-breaking lagrangian, $v=\left(2 \sqrt{2} G_{F}\right)^{-1 / 2}, \tan \beta$ and $s$, doublet and singlet vacuum expectation values or related quantities. Here, we have been considering the $Z_{3^{-}}$ conserving NMSSM explicitly. However, $Z_{3}$-violating terms as well as radiative corrections can be incorporated in this picture with limited effort. The states $A_{i}=P_{i 1} A^{0}+P_{i 2} A_{S}^{0}$ 
are ordered in mass: $m_{A_{1}}<m_{A_{2}}$. A light state is regarded as natural - i.e. as a pseudoNambu-Goldstone boson - in two specific limits of the NMSSM parameter space:

- For $\kappa \ll \lambda$, the Higgs potential is approximately invariant under a U(1) Peccei-Quinn symmetry. In this case, the doublet component of $A_{1}$ - through which this particle couples to SM matter - is given by $P_{11}=-\frac{v}{2 s} \sin 2 \beta / \sqrt{1+\frac{v^{2}}{4 s^{2}} \sin ^{2} 2 \beta}$.

- For $A_{\lambda}, A_{\kappa} \rightarrow 0$, another approximate $\mathrm{U}(1)$ symmetry appears, which can be related to the R-symmetry. In this case, $P_{11}=\frac{v}{s} \sin 2 \beta / \sqrt{1+\frac{v^{2}}{s^{2}} \sin ^{2} 2 \beta}$.

However, the light pseudoscalar may also result from an 'accidental' arrangement of the NMSSM parameters.

While a MSSM pseudoscalar might still be comparatively light [2] (see also [3] for a discussion in the context of the NMSSM), the hypothesis of a dominantly doublet light CP-odd Higgs - already constrained (at least indirectly) by LEP [4] — is under increasing pressure from LHC searches $[5,6]$ or flavour transitions, due to the correlation of doublet masses - implying that there exist a light CP-even and a comparatively light pair of charged Higgs states as well, all phenomenologically more conspicuous at colliders. Still, the situation is different for a singlet or a mixed pseudoscalar $A_{1}$, since the mentioned correlation dissipates and the light $\mathrm{CP}$-odd Higgs becomes largely independent from the rest of the Higgs sector. From the perspective of LEP (or $e^{+} e^{-}$-colliders in general), the direct production of a CP-odd Higgs proves difficult as the tree-level couplings to electroweak gauge-bosons vanish. The possibility of a light CP-odd NMSSM state - with mass $\lesssim 10 \mathrm{GeV}$ - thus appeared in the pre-LHC era as a phenomenologically appealing and realistic scenario: see e.g. [7-10]. Nevertheless, limits from low-energy observables, e.g. flavour transitions [11-15] or bottomonium decays and spectroscopy [16-29], apply in this low-mass region and constrain, in particular, the coupling of $A_{1}$ to down-type fermions. With the start of the LHC, several direct or indirect production modes of the light CP-odd Higgs have been considered [30-35]. Yet, the Higgs discovery at the LHC [36, 37] considerably reduces the scope of the phenomenology associated to a light $A_{1}$ : when kinematically allowed, the Higgs-to-Higgs decay $H[125] \rightarrow 2 A_{1}$ - with $H[125]$ denoting the observed state at $\sim 125 \mathrm{GeV}$ - could naively dominate the standard decay channels, which would have implied suppressed rates of $H[125]$ in the Run-I (and Run-II). As the observed Higgs characteristics demonstrate the success of the standard search channels, the $H[125] \rightarrow 2 A_{1}$ decay width must therefore be small. This can be realized — in certain limits or due to accidental cancellations - and, in this extent, a light NMSSM $A_{1}$ may coexist with a CP-even state at $\sim 125 \mathrm{GeV}$ that retains roughly SM-like characteristics hence a suitable candidate for $H[125]$. Such a scenario thus remains phenomenologically viable. Nevertheless, the condition of a suppressed $H[125] \rightarrow 2 A_{1}$ induces constraints on the NMSSM parameter space, which have been discussed in e.g. [3, 38]. Additionally, ATLAS and CMS have searched explicitly for $H[125] \rightarrow 2 A_{1}$ with final states including leptons [39-41].

Beyond its consequences for the Higgs sector, the hypothetical existence of a light NMSSM pseudoscalar may lead to other phenomenologically interesting effects. In the 
context of singlino Dark Matter [42-45], the light- $A_{1}$ funnel may ensure a sufficiently large annihilation cross-section, yielding the correct relic density [46-52]. In this sense, the light CP-odd Higgs scenario retains a clear motivation. Another application [53] would address the $17 \mathrm{MeV}$ excess in ${ }^{8} \mathrm{Be}$ transitions [54].

Despite the interest that the light CP-odd Higgs scenario has raised in the literature, a relative shadow continues to veil our knowledge of the decays of this particle in the very low mass range $m_{A_{1}} \lesssim 2 m_{\tau}$. There, the partonic description, summarized in e.g. [28], predicts largely dominant strongly-interacting final states, such as $g g$ or $s \bar{s}$. This picture has been sensibly criticized by [55]: close to the confinement scale, the partonic approach is no longer reliable and [55] recommends an effective description of the hadronic decays based on the perturbative spectator model. Among the consequences of the latter choice, $m_{A_{1}}=3 m_{\pi^{0}} \sim 0.4 \mathrm{GeV}$ becomes the lower limit where hadronic final states are relevant. Moreover, hadronic channels then seem largely superseeded by the $A_{1} \rightarrow \mu^{+} \mu^{-}$width. Still, this description in [55] misses at least one effect that can substantially affect the decays: the CP-odd Higgs shares its quantum numbers with (some of) the mesons, which induces a mixing among these states. In other words, the light CP-odd Higgs acquires a mesonic component — via its interaction with quarks - and the latter may well dominate the decays of this particle. In this sense, the impact of hadronic physics extends below the tri-pion threshold, at least down to $m_{A_{1}} \sim m_{\pi^{0}}$. This mixing effect has already been noted in the context of heavy quarkonia $[16,27]$ and its impact on $A_{1}$-decays at the $b \bar{b}$-threshold was highlighted in [56]. In the very low-mass range, [57] suggested that the hadronic decays of $A_{1}$ may resemble those of the meson that is closest in mass, while [58] estimated the mixing with the mesons in the formalism of Partially-Conserved Axial Currents (PCAC). As a consequence of this confused situation for the pseudoscalar decays, the phenomenology of this particle at low-mass remains largely speculative and the interplay of constraints cannot be consistently applied.

In this paper, we aim at shedding some light into this question and propose an estimate of the NMSSM pseudoscalar decays in the $m_{A_{1}} \lesssim 3 \mathrm{GeV}$ range. Due to the intrinsic difficulty of a quantitative description of hadronic phenomena and the corresponding large uncertainties, this evaluation has no ambition beyond that of providing an educated guess for the $A_{1}$ decay widths and branching fractions and, while the derived picture may seem more reliable than the partonic approach, we should not dismiss the possibility of sizable deviations. In the following section, we shall summarize the formalism describing the interactions of a light CP-odd Higgs with the mesons, relevant at masses below $\lesssim 1 \mathrm{GeV}$. Then, we will derive the $A_{1}$ decays in this mass range. Finally, we will attempt to extrapolate the hadronic decays of the pseudoscalar up to the $c \bar{c}$ threshold using the perturbative spectator approach, before coming to a short conclusion.

As a final word before starting with the actual description of the pseudoscalar interactions at low mass, we stress that our results apply beyond the NMSSM, in any singlet or doublet extension of the SM containing a light pseudoscalar Higgs state: all that is necessary in order to extend our discussion to such cases amounts to replacing the explicit NMSSM couplings to quarks, photons and gluons by their analogues in the corresponding model. 


\section{From the partonic lagrangian to the mesonic interactions}

The purpose of this section consists in summarizing the formalism leading to the inclusion of a light pseudoscalar in the non-linear Sigma model for the mesons.

\subsection{Partonic lagrangian below the $c \bar{c}$ threshold}

We consider a NMSSM CP-odd Higgs with mass below the $c \bar{c}$ threshold. The other relevant fields at low-energy include the up, down and strange quarks, the muon and electron, as well as the photon and gluons. The interactions of the pseudoscalar with these fields may be summarized in the following effective lagrangian:

$$
\begin{aligned}
\mathcal{L}_{A_{1}}= & \frac{\imath P_{11}}{\sqrt{2} v} A_{1}\left\{m_{u} \tan ^{-1} \beta \bar{u} \gamma_{5} u+m_{d} \tan \beta \bar{d} \gamma_{5} d+m_{s} \tan \beta \bar{s} \gamma_{5} s+m_{\mu} \tan \beta \bar{\mu} \gamma_{5} \mu+m_{e} \tan \beta \bar{e} \gamma_{5} e\right\} \\
& +\frac{\alpha}{4 \pi} C_{\gamma} A_{1} F_{\mu \nu} \tilde{F}^{\mu \nu}+\frac{\alpha_{s}}{4 \pi} C_{g} A_{1} G_{\mu \nu}^{a} \tilde{G}^{a \mu \nu}
\end{aligned}
$$

Here, we have confined to the operators of lowest-dimension for the $A_{1}$-interactions with each type of field: dimension 4 for the fermions and dimension 5 for the gauge bosons. We have kept the tree-level expression of the fermionic couplings, though part of the radiative corrections may be incorporated within $P_{11}$ - defined by eq. (1.1) and corresponding to the proportion of doublet-component in $A_{1} . F_{\mu \nu}$ and $G_{\mu \nu}^{a}$ denote the field-strength tensors for the photonic and gluonic fields respectively; $\tilde{F}_{\mu \nu}$ and $\tilde{G}_{\mu \nu}^{a}$ are their dual.

The couplings $C_{\gamma}$ and $C_{g}$ are generated by loops of heavy fermions $(t, b, \tau, c$ and charginos); heavy scalars and gauge bosons are known not to contribute, due to nonrenormalization theorems. Since we regard the light quarks and leptons as 'active' fields, we do not include their radiative contribution in $C_{\gamma}$ and $C_{g}$ : for the leptons, this effect could be added straightforwardly; in the case of light quark contributions, however, inclusion at the partonic level should be reputed unreliable. At the one-loop level:

$$
\begin{aligned}
C_{\gamma}= & -\frac{P_{11}}{2 \sqrt{2} v}\left\{\frac{N_{c} Q_{u}^{2}}{\tan \beta}\left[\mathcal{F}\left(\frac{m_{t}^{2}}{m_{A_{1}}^{2}}\right)+\mathcal{F}\left(\frac{m_{c}^{2}}{m_{A_{1}}^{2}}\right)\right]+N_{c} Q_{d}^{2} \tan \beta \mathcal{F}\left(\frac{m_{b}^{2}}{m_{A_{1}}^{2}}\right)+Q_{e}^{2} \tan \beta \mathcal{F}\left(\frac{m_{\tau}^{2}}{m_{A_{1}}^{2}}\right)\right\} \\
& -\frac{Q_{\chi}^{2}}{2 \sqrt{2}} \sum_{i=1}^{2} \frac{1}{m_{\chi_{i}^{ \pm}}}\left[\lambda P_{12} U_{i 2} V_{i 2}-g P_{11}\left(\cos \beta U_{i 1} V_{i 2}+\sin \beta U_{i 2} V_{i 1}\right)\right] \mathcal{F}\left(\frac{m_{\chi_{i}^{ \pm}}^{2}}{m_{A_{1}}^{2}}\right) \\
C_{g}= & -\frac{P_{11}}{4 \sqrt{2} v}\left\{\frac{1}{\tan \beta}\left[\mathcal{F}\left(\frac{m_{t}^{2}}{m_{A_{1}}^{2}}\right)+\mathcal{F}\left(\frac{m_{c}^{2}}{m_{A_{1}}^{2}}\right)\right]+\tan \beta \mathcal{F}\left(\frac{m_{b}^{2}}{m_{A_{1}}^{2}}\right)\right\} \\
N_{c}= & 3 ; \quad Q_{u}=\frac{2}{3} ; \quad Q_{d}=-\frac{1}{3} ; Q_{e}=Q_{\chi}=-1 ; \mathcal{F}(x)=2 x \log ^{2}\left[\frac{\sqrt{1-4 x}-1}{\sqrt{1-4 x}+1}\right]
\end{aligned}
$$

Beyond the obvious notations for the SM fermions, we have introduced the chargino masses $m_{\chi_{i}^{ \pm}}$and diagonalizing matrices $U, V$ : we refer the reader to appendix A of [1] for the details of the conventions. We remind that the logarithm in the definition of $\mathcal{F}$ is taken in its complex sense.

Finally, we define the axial currents associated to the light quarks:

$$
J_{A}^{a \mu}=(\bar{q})^{T} \gamma^{\mu} \gamma_{5} \lambda^{a}(q) ; \quad(q) \equiv(u, d, s)^{T}
$$

where $\lambda^{a}$ are the Gell-Mann matrices acting in flavour space - in particular $\lambda^{3} \equiv$ $\operatorname{diag}(1,-1,0) / \sqrt{2}, \lambda^{8} \equiv \operatorname{diag}(1,1,-2) / \sqrt{6}$ and $\lambda^{9} \equiv \operatorname{diag}(1,1,1) / \sqrt{3}$ - and normalized 
to $\operatorname{Tr}\left[\lambda^{a} \lambda^{b}\right]=\delta^{a b}$. We note that the $A_{1}$ couplings to the light quarks may be related to the divergences of the neutral currents [58]:

$$
\begin{aligned}
\mathcal{L}_{A_{1}} \ni \frac{P_{11}}{4 v} A_{1}\left\{\left(\tan ^{-1} \beta-\tan \beta\right) \partial_{\mu} J_{A}^{3 \mu}+\right. & \frac{1}{\sqrt{3}}\left(\tan ^{-1} \beta-\tan \beta\right) \partial_{\mu} J_{A}^{8 \mu} \\
& \left.+\sqrt{\frac{2}{3}}\left(\tan ^{-1} \beta+2 \tan \beta\right) \partial_{\mu} J_{A}^{9 \mu}\right\}+\ldots
\end{aligned}
$$

We also remind the coupling of the photon and gluon to the chiral anomalies:

$$
\begin{aligned}
\partial_{\mu} J_{A}^{a \mu} & =\imath(\bar{q})^{T} \gamma_{5}\left\{\lambda^{a}, m_{q}\right\}(q)+\frac{\alpha_{s}}{4 \pi} \operatorname{Tr}\left[\lambda^{a}\right] G_{\mu \nu}^{a} \tilde{G}^{a \mu \nu}+\frac{2 \alpha N_{c}}{4 \pi} \operatorname{Tr}\left[\lambda^{a} Q_{q}^{2}\right] F_{\mu \nu} \tilde{F}^{\mu \nu} \\
\partial_{\mu} J_{A}^{3 \mu} & =\sqrt{2} \imath\left[m_{u} \bar{u} \gamma_{5} u-m_{d} \bar{d} \gamma_{5} d\right]+\frac{\alpha}{4 \pi} \sqrt{2} F_{\mu \nu} \tilde{F}^{\mu \nu} \\
\partial_{\mu} J_{A}^{8 \mu} & =\sqrt{\frac{2}{3}} \imath\left[m_{u} \bar{u} \gamma_{5} u+m_{d} \bar{d} \gamma_{5} d-2 m_{s} \bar{d} \gamma_{5} s\right]+\frac{\alpha}{4 \pi} \sqrt{\frac{2}{3}} F_{\mu \nu} \tilde{F}^{\mu \nu} \\
\partial_{\mu} J_{A}^{9 \mu} & =\frac{2}{\sqrt{3}} \imath\left[m_{u} \bar{u} \gamma_{5} u+m_{d} \bar{d} \gamma_{5} d+m_{s} \bar{d} \gamma_{5} s\right]+\frac{\alpha_{s}}{4 \pi} \sqrt{3} G_{\mu \nu}^{a} \tilde{G}^{a \mu \nu}+\frac{\alpha}{4 \pi} \frac{4}{\sqrt{3}} F_{\mu \nu} \tilde{F}^{\mu \nu}
\end{aligned}
$$

with $m_{q}=\operatorname{diag}\left(m_{u}, m_{d}, m_{s}\right)$ and $Q_{q}=\operatorname{diag}\left(\frac{2}{3},-\frac{1}{3},-\frac{1}{3}\right)$ the quark mass and charge matrices.

\subsection{Chiral lagrangian}

The dynamics of the mesons is well described - at lowest order in a momentum expansion - by a non-linear sigma model known as the Chiral Perturbation Theory ( $\chi \mathrm{PT})$ [59-63]. This formalism relies on a controlled breaking of the axial symmetries and proves remarkably predictive. Though refinements including higher-dimension terms are possible [63], they lead to a fast increase of the number of free low-energy parameters and we shall confine to the simplest approach below. A recurrent endeavour of the 1980's consisted in estimating the couplings of a hypothetically light SM Higgs boson — or a 2HDM CP-even state - to the hadronic sector [64-76]. Some attention was also paid to the case of a pseudoscalar [77, 78], and has persisted till today at least from the perspective of axion physics (see e.g. [79] for a recent reference). In the following, we aim at summarizing the key ingredients that intervene in the description of the interactions of a light CP-odd Higgs with the meson sector.

The starting point of $\chi \mathrm{PT}$ rests with the observation that the QCD lagrangian for the light quarks $(q)$ preserves the axial symmetry - characterized by the transformation $(q) \mapsto$ $U\left[\alpha_{a}\right](q)$, with $U\left[\alpha_{a}\right] \equiv \exp \left[\imath \alpha_{a} \lambda^{a} \gamma_{5}\right]$ - up to the mass term $M_{q}$ and the electromagnetic interaction. In our case, the Yukawa couplings to the light CP-odd Higgs - see eq. (2.1) - can be incorporated within the mass matrix:

$$
\begin{aligned}
\mathcal{L}_{q} & =(\bar{q})^{T}\left\{\imath \gamma^{\mu} D_{\mu}-M_{q}\left[A_{1}\right]\right\}(q) ; \quad D_{\mu}(q) \equiv\left(\partial_{\mu}-\imath g_{s} T^{a} G_{\mu}^{a}-\imath e Q_{q} A_{\mu}\right)(q) \\
M_{q}\left[A_{1}\right] & \equiv \operatorname{diag}\left[m_{u}\left(1-\frac{\imath P_{11}}{\sqrt{2} v \tan \beta} A_{1}\right), m_{d}\left(1-\frac{\imath P_{11} \tan \beta}{\sqrt{2} v} A_{1}\right), m_{s}\left(1-\frac{\imath P_{11} \tan \beta}{\sqrt{2} v} A_{1}\right)\right]
\end{aligned}
$$


with $g_{s}$ the strong coupling constant, $T^{a}$ the Gell-Mann matrices in colour-space, $e$ the elementary electric charge, $Q_{q}$ the quark-charge matrix (as defined above), $G_{\mu}^{a}$ the gluon field and $A_{\mu}$ the photon field.

Since the strong interaction triggers the formation of quark condensates at low energy, we shift our attention from the fundamental 3 to the $\overline{3} \times 3$ representations of $\mathrm{U}(3)_{\text {flavour }}$, the mesonic octet and singlet $\Sigma_{i j} \sim\left\langle\bar{q}_{i} \gamma_{5} q_{j}\right\rangle$. Then, the lowest-order effective lagrangian for $\Sigma$ preserving the axial (and vectorial) symmetry up to $M_{q}$ and $Q_{q}$ reads:

$$
\mathcal{L}_{\chi}=\operatorname{Tr}\left\{D_{\mu} \Sigma D^{\mu} \Sigma^{\dagger}+\frac{B}{2}\left[M_{q}\left[A_{1}\right] \Sigma+\Sigma^{\dagger} M_{q}\left[A_{1}\right]^{\dagger}\right]\right\}+\frac{C}{2}\left(\partial_{\mu} K^{\mu}\right)^{2}+\imath \partial_{\mu} K^{\mu}\left[\frac{1}{2} \operatorname{Tr} \log \Sigma-\imath C_{g} A_{1}\right]
$$

Here, $D_{\mu} \Sigma \equiv \partial_{\mu} \Sigma-\imath e A_{\mu}\left[Q_{q}, \Sigma\right], B$ and $C$ are coupling constants and $\partial_{\mu} K^{\mu} \sim \frac{\alpha_{s}}{4 \pi} G_{\mu \nu}^{a} \tilde{G}^{a \mu \nu}$ is an auxilliary field designed to mimic the gluon coupling to the $\mathrm{U}(1)_{A}$ anomaly [59]. In other words, considering the axial currents $\mathcal{J}_{\mu}^{a} \equiv \imath \operatorname{Tr}\left\{\partial_{\mu} \Sigma\left\{\lambda^{a}, \Sigma^{\dagger}\right\}-\left\{\lambda^{a}, \Sigma\right\} \partial_{\mu} \Sigma^{\dagger}\right\}$, one obtains:

$$
\partial_{\mu} \mathcal{J}^{9 \mu} \ni \sqrt{3} \partial_{\mu} K^{\mu} \sim \frac{\sqrt{3} \alpha_{s}}{4 \pi} G_{\mu \nu}^{a} \tilde{G}^{a \mu \nu}
$$

Similarly, the gluonic coupling of $A_{1}$ is accounted for in eq. (2.7) by the $\partial_{\mu} K^{\mu}$ term. The minimization condition for $\partial_{\mu} K^{\mu}$ provides the chiral lagrangian:

$$
\tilde{\mathcal{L}}_{\chi}=\operatorname{Tr}\left\{D_{\mu} \Sigma D^{\mu} \Sigma^{\dagger}+\frac{B}{2}\left[M_{q}\left[A_{1}\right] \Sigma+\Sigma^{\dagger} M_{q}\left[A_{1}\right]^{\dagger}\right]\right\}-\frac{1}{2 C}\left[C_{g} A_{1}+\frac{\imath}{2} \operatorname{Tr} \log \Sigma\right]^{2}
$$

We finally introduce the pion fields $\pi_{a}$ as $\Sigma \equiv \frac{f_{\pi}}{2} \exp \left[\frac{\imath \sqrt{2}}{f_{\pi}} \pi_{a} \lambda^{a}\right]$ and expand the lagrangian in terms of these:

$$
\begin{aligned}
& \tilde{\mathcal{L}}_{\chi} \simeq \frac{1}{2}\left\{D_{\mu} \pi_{a} D^{\mu} \pi_{a}+\frac{B P_{11}}{v} A_{1} \pi_{a} \operatorname{Tr}\left[\lambda^{a} \tilde{M}_{q}\right]-\frac{B}{f_{\pi}} \pi_{a} \pi_{b} \operatorname{Tr}\left[\lambda^{a} \lambda^{b} m_{q}\right]\right. \\
& \left.-\frac{B P_{11}}{3 v f_{\pi}^{2}} A_{1} \pi_{a} \pi_{b} \pi_{c} \operatorname{Tr}\left[\lambda^{a} \lambda^{b} \lambda^{c} \tilde{M}_{q}\right]+\frac{B}{6 f_{\pi}^{3}} \pi_{a} \pi_{b} \pi_{c} \pi_{d} \operatorname{Tr}\left[m_{q} \lambda^{a} \lambda^{b} \lambda^{c} \lambda^{d}\right]-\frac{1}{C}\left[C_{g} A_{1}-\frac{1}{f_{\pi}} \sqrt{\frac{3}{2}} \pi_{9}\right]^{2}\right\}+\ldots
\end{aligned}
$$

where $m_{q} \equiv M_{q}[0]$ and $\tilde{M}_{q} \equiv \operatorname{diag}\left[\frac{m_{u}}{\tan \beta}, m_{d} \tan \beta, m_{s} \tan \beta\right]$. We observe that this procedure generates mass terms for the mesons, a mass shift for $A_{1}$, mixing terms between the mesons and $A_{1}$ as well as quartic interaction terms involving $A_{1}$ and three pions - the conservation of $\mathrm{CP}$ excludes a cubic coupling. Our derivation of the decays of the light pseudoscalar will be based on this simple lagrangian. Using the pion equations of motion, it is possible to check that, as in the quark model, the couplings of $A_{1}$ to the hadronic sector follow the PCAC, i.e.

$$
\begin{gathered}
\tilde{\mathcal{L}}_{\chi} \ni \frac{P_{11} A_{1}}{4 v}\left\{\left(\tan ^{-1} \beta-\tan \beta\right) \partial^{\mu} \mathcal{J}_{\mu}^{3}+\frac{1}{\sqrt{3}}\left(\tan ^{-1} \beta-\tan \beta\right) \partial^{\mu} \mathcal{J}_{\mu}^{8}\right. \\
\left.+\sqrt{\frac{2}{3}}\left(\tan ^{-1} \beta+2 \tan \beta\right) \partial^{\mu} \mathcal{J}_{\mu}^{9}\right\}
\end{gathered}
$$

This could have been chosen as an equivalent Ansatz for the $A_{1}$ interactions. Yet, the previous formalism has allowed us to include the $A_{1}$ coupling to gluons in the low-energy picture as well. 
So far we have omitted Wess-Zumino-Witten terms describing the pion coupling to photons $[80,81]$ - and restoring the corresponding contribution to the anomaly:

$$
\mathcal{L}_{W Z W} \simeq-\frac{\sqrt{2} N_{c} \alpha}{4 \pi f_{\pi}} \pi_{a} \operatorname{Tr}\left[Q_{q}^{2} \lambda^{a}\right] F_{\mu \nu} \tilde{F}^{\mu \nu}+\ldots
$$

We now add this piece to eq. (2.10).

\subsection{Low-energy coupling constants - meson masses}

The low-energy lagrangian of eq. (2.10) has left us with five combinations of couplings to determine at low-energy: $\frac{B m_{u}}{f_{\pi}}, \frac{B m_{d}}{f_{\pi}}, \frac{B m_{s}}{f_{\pi}}, f_{\pi}, C . f_{\pi} \simeq 93 \mathrm{MeV}$ is the pion decay constant: it determines the pion coupling to the anomaly, hence its diphoton decay, from which it is extracted. The other parameters are usually obtained from the mass matrix of the mesons: from eq. (2.10), $\left[\mathcal{M}_{\pi}^{2}\right]^{a b}=\frac{B}{f_{\pi}} \operatorname{Tr}\left[\lambda^{a} \lambda^{b} m_{q}\right]+\frac{3}{2 f_{\pi}^{2} C} \delta^{a 9} \delta^{b 9}$. We may thus identify:

- three pions with diagonal mass $m_{\pi}^{2} \equiv \frac{B}{2 f_{\pi}}\left(m_{u}+m_{d}\right) \simeq(135 \mathrm{MeV})^{2}$; the electromagnetic interaction generates an additional mass contribution to the charged pions $\pi^{ \pm}=\frac{\pi_{1} \mp \imath \pi_{2}}{\sqrt{2}}$; in principle, the neutral pion $\pi_{3}$ mixes with other neutral states $\left(\pi_{8}\right.$, $\pi_{9}$ ), but the mixing terms $\propto m_{u}-m_{d}$ are numerically small so that we may neglect them at this level. Thus $\frac{B m_{u}}{f_{\pi}} \simeq \frac{B m_{d}}{f_{\pi}} \simeq m_{\pi}^{2}$.

- a pair of charged kaons $K^{ \pm}=\frac{\pi_{4} \mp l \pi_{5}}{\sqrt{2}}$ with mass $m_{K^{ \pm}}^{2}=\frac{B}{2 f_{\pi}}\left(m_{u}+m_{s}\right) \simeq(494 \mathrm{MeV})^{2}$ and a pair of neutral kaons $K^{0}, \bar{K}^{0}=\frac{\pi_{6} \mp \imath \pi_{7}}{\sqrt{2}}$ with mass $m_{K^{0}}^{2}=\frac{B}{2 f_{\pi}}\left(m_{d}+m_{s}\right) \simeq$ $(498 \mathrm{MeV})^{2}$. Therefore, $\frac{B m_{s}}{f_{\pi}} \simeq m_{K^{0}}^{2}+m_{K^{ \pm}}^{2}-m_{\pi}^{2}$.

- The neutral $\pi_{8}$ and $\pi_{9}$ mix according to the following matrix:

$$
\begin{aligned}
{\left[\begin{array}{cc}
m_{\pi_{8}}^{2} & \Delta \\
\Delta & m_{\pi_{9}}^{2}
\end{array}\right] ; \quad m_{\pi_{8}}^{2} } & \equiv \frac{B}{6 f_{\pi}}\left(m_{u}+m_{d}+4 m_{s}\right) \\
m_{\pi_{9}}^{2} & \equiv \frac{B}{3 f_{\pi}}\left(m_{u}+m_{d}+m_{s}\right)+\frac{3}{2 f_{\pi}^{2} C} \\
\Delta & \equiv \frac{B}{3 \sqrt{2} f_{\pi}}\left(m_{u}+m_{d}-2 m_{s}\right)
\end{aligned}
$$

The corresponding mass states are the $\eta=\cos \theta_{\eta} \pi_{8}-\sin \theta_{\eta} \pi_{9}$ and $\eta^{\prime}=\sin \theta_{\eta} \pi_{8}+$ $\cos \theta_{\eta} \pi_{9}$, with masses of $m_{\eta} \simeq 548 \mathrm{MeV}$ and $m_{\eta^{\prime}} \simeq 958 \mathrm{MeV}$ and a mixing angle $\theta_{\eta}$ of order $-13^{\circ}$ (see e.g. [82-84]). The values of $m_{\pi_{8}}^{2} \simeq(575 \mathrm{MeV})^{2}$ and $\Delta \simeq$ $-(370 \mathrm{MeV})^{2}$ are in rough agreement with what we could expect in view of the pion and kaon masses. $m_{\pi_{9}}^{2} \simeq(940 \mathrm{MeV})^{2}$ provides $\frac{3}{2 C f_{\pi}^{2}} \simeq m_{\pi_{9}}^{2}-\frac{1}{3}\left(m_{K^{0}}^{2}+m_{K^{ \pm}}^{2}+m_{\pi}^{2}\right) \simeq$ $m_{\pi_{9}}^{2}-\frac{1}{2}\left(m_{\pi_{8}}^{2}+m_{\pi}^{2}\right) \simeq(850 \mathrm{MeV})^{2}$

This fully determines the low-energy parameters that we employ in the following. 


\section{Decays of a CP-odd Higgs from the chiral lagrangian}

The chiral lagrangian of eq. (2.10) that we have derived in the previous section characterizes the interactions of the Higgs pseudoscalar with the mesons. At low masses $m_{A_{1}} \lesssim 1 \mathrm{GeV}$, it should prove a reliable guide for the hadronic decays of the CP-odd Higgs. Additionally, the leptonic and photonic interactions can be taken directly from eq. (2.1). In this section, we derive the associated phenomenology in this low-mass regime. In order to smoothen the transition with the higher-mass region, we shall display the Higgs decays up to $m_{A_{1}} \sim$ $1.5 \mathrm{GeV}$ in numerical applications.

\section{$3.1 \quad A_{1}$-meson mixing}

eq. (2.10) shows that the interactions of the pseudoscalar with the hadronic sector generate a mixing of $A_{1}$ with $\pi_{3}, \pi_{8}$ and $\pi_{9}$ :

$$
\begin{gathered}
\tilde{\mathcal{L}}_{\chi} \ni-\frac{1}{2}\left(\pi_{3}, \pi_{8}, \pi_{9}, A_{1}\right) \mathcal{M}_{\operatorname{mix}}^{2}\left(\begin{array}{l}
\pi_{3} \\
\pi_{8} \\
\pi_{9} \\
A_{1}
\end{array}\right) ; \quad \mathcal{M}_{\operatorname{mix}}^{2}=\left[\begin{array}{cccc}
m_{\pi}^{2} & \frac{1}{\sqrt{3}} \delta & \sqrt{\frac{2}{3}} \delta & \delta m_{3}^{2} \\
\frac{1}{\sqrt{3}} \delta & m_{\pi_{8}}^{2} & \Delta & \delta m_{8}^{2} \\
\sqrt{\frac{2}{3}} \delta & \Delta & m_{\pi_{9}}^{2} & \delta m_{9}^{2} \\
\delta m_{3}^{2} & \delta m_{8}^{2} & \delta m_{9}^{2} & \bar{m}_{A_{1}}^{2}
\end{array}\right] \\
\left\{\begin{array}{l}
\delta \equiv \frac{B}{2 f_{\pi}}\left(m_{u}-m_{d}\right) \simeq 0 \\
\delta m_{3}^{2} \equiv-\frac{B P_{11}}{2 \sqrt{2} v}\left(\frac{m_{u}}{\tan \beta}-m_{d} \tan \beta\right)=-\frac{f_{\pi} P_{11}}{2 \sqrt{2} v}\left[m_{\pi}^{2}\left(\tan ^{-1} \beta-\tan \beta\right)+\delta\left(\tan ^{-1} \beta+\tan \beta\right)\right] \\
\delta m_{8}^{2} \equiv-\frac{B P_{11}}{2 \sqrt{6} v}\left(\frac{m_{u}}{\tan \beta}+m_{d} \tan \beta-2 m_{s} \tan \beta\right) \\
\quad=-\frac{f_{\pi} P_{11}}{2 \sqrt{2} v}\left[-\sqrt{3} \tan \beta m_{\pi_{8}}^{2}+\frac{m_{\pi}^{2}}{\sqrt{3}}\left(\tan ^{-1} \beta+2 \tan \beta\right)+\frac{\delta}{\sqrt{3}}\left(\tan ^{-1} \beta-\tan \beta\right)\right] \\
\delta m_{9}^{2} \equiv-\frac{B P_{11}}{2 \sqrt{3} v}\left(\frac{m_{u}}{\tan \beta}+m_{d} \tan \beta+m_{s} \tan \beta\right)-\sqrt{\frac{3}{2} \frac{C_{g}}{f_{\pi} C}} \\
=-\frac{f_{\pi} P_{11}}{2 \sqrt{2} v}\left[\sqrt{\frac{3}{2}} \tan \beta m_{\pi_{8}}^{2}+\frac{m_{\pi}^{2}}{\sqrt{6}}\left(2 \tan ^{-1} \beta+\tan \beta\right)+\sqrt{\frac{2}{3}} \delta\left(\tan ^{-1} \beta-\tan \beta\right)\right] \\
\quad-\sqrt{\frac{2}{3}} f_{\pi} C_{g}\left[m_{\pi_{9}}^{2}-\frac{1}{3}\left(m_{\pi_{8}}^{2}+m_{\pi}^{2}\right)\right]
\end{array}\right. \\
\bar{m}_{A_{1}}^{2}=m_{A_{1}}^{2}+\frac{1}{C} C_{g}^{2}
\end{gathered}
$$

We note that the mass-shift of $A_{1}$ resulting from the gluon coupling $-\frac{1}{C} C_{g}^{2}-$ is typically tiny: sub-MeV'.

The diagonalization of $\mathcal{M}_{\text {mix }}^{2}=O \cdot \operatorname{diag}\left[\tilde{m}_{\pi}^{2}, \tilde{m}_{\eta}^{2}, \tilde{m}_{\eta^{\prime}}^{2}, \tilde{m}_{A_{1}}^{2}\right] \cdot O^{T}$ - where $O$ is the orthogonal transition matrix - defines the mass-states $\left(\tilde{\pi}^{0}, \tilde{\eta}, \tilde{\eta}^{\prime}, \tilde{A}_{1}\right)^{T}=O \cdot\left(\pi_{3}, \pi_{8}, \pi_{9}, A_{1}\right)^{T}$. If the presence of the Higgs pseudoscalar were to affect drastically the characteristics of the pions, then this mixing scenario would be phenomenologically difficult to defend. In practice, however, the mixing is naturally small. We note indeed that the off-diagonal elements of $\mathcal{M}_{\text {mix }}^{2}$ are suppressed by a prefactor $\frac{f_{\pi}}{v} \sim 5 \cdot 10^{-4}$. Moreover, they explicitly involve the doublet component of $A_{1}-P_{11}$ controls the interactions of the pseudoscalar with SM-matter - , which tends to be suppressed, i.e. $P_{11}^{2} \ll 1$ in the realistic cases: the light $A_{1}$ is likely to intervene as a dominantly singlet state. Consequently, the $A_{1}$-meson mixing has a negligible impact on the mesons. In [58], for instance, it was shown that the pseudoscalar could approach the pion mass within $\mathrm{MeV}$ wihout affecting the pion decays as 
long as the $A_{1}-\pi_{3}$ mixing remained below $\sim 4 \%$. Similarly, the mass-shift associated with the mixing remains tiny. Another type of limit proceeds from the conversion of hadrons to $A_{1}$ via mixing. For instance, $K^{+} \rightarrow \pi^{+} \pi^{0}$ induces $K^{+} \rightarrow \pi^{+} A_{1}$. In the case of a sizable $A_{1} \rightarrow$ invisible decay, the $K^{+} \rightarrow \pi^{+} \nu \bar{\nu}$ searches would place a limit on the $A_{1}-\pi_{3}$ mixing. Similar bounds proceed from leptonic decays (or leptonic transitions mediated by an off-shell $A_{1}$ ). The interference with loop-generated flavour-changing $A_{1}$ couplings would have to be considered, however. Additionally, experimental cuts require some attention as e.g. the vicinity of the pion mass is occasionally discarded in order to avoid the pion background. We shall not enter into a detailed discussion here as the $A_{1}$ decays need first be derived. We may thus safely assume $\tilde{\pi}^{0} \sim \pi_{3}, \tilde{\eta} \sim \eta$ and $\tilde{\eta}^{\prime} \sim \eta^{\prime}$, although a narrower check would be in order when $m_{A_{1}}$ is very near a meson mass. We shall discuss this further when we compute the leptonic decay widths. On the other hand, the impact of the mixing on the phenomenology of $A_{1}$ is sizable because the couplings of this field to SM particles are suppressed in the same proportion as the mixing: in other words, even a small meson component in $\tilde{A}_{1}$ could dominate the genuine $A_{1}$ amplitudes. In the following, we thus focus on the corresponding state: ${ }^{1}$

$$
\tilde{A}_{1}=O_{A 3} \pi_{3}+O_{A 8} \pi_{8}+O_{A 9} \pi_{9}+O_{A A} A_{1}=O_{A 3} \pi_{3}+O_{A \eta} \eta+O_{A \eta^{\prime}} \eta^{\prime}+O_{A A} A_{1}
$$

In the limit where the mixing angles remain small — which is almost systematically fulfiled and ensures that the mesons do not receive a sizable Higgs component - , these matrix elements can be approximated by:

$$
\begin{aligned}
O_{A A} & \simeq 1 & O_{A 3} & \simeq \frac{\delta m_{3}^{2}}{m_{A_{1}}^{2}-m_{\pi}^{2}} \\
O_{A \eta} & \simeq \frac{\cos \theta_{\eta} \delta m_{8}^{2}-\sin \theta_{\eta} \delta m_{9}^{2}}{m_{A_{1}}^{2}-m_{\eta}^{2}} & O_{A \eta^{\prime}} & \simeq \frac{\sin \theta_{\eta} \delta m_{8}^{2}+\cos \theta_{\eta} \delta m_{9}^{2}}{m_{A_{1}}^{2}-m_{\eta^{\prime}}^{2}}
\end{aligned}
$$

Nevertheless, in numerical applications, we will retain the full numerical evaluation of these objects.

We plot these mixing angles in figure 1 for $P_{11}=0.03, \tan \beta=10$. The mixings prove very small (below $\sim 10^{-8}$ ) on the whole mass-range, except when $m_{A_{1}}$ is in the immediate vicinity of a meson mass. The impact of the mixing on the mesonic state will thus remain negligible.

Then, any decay amplitude of the pseudoscalar may be decomposed as:

$$
\mathcal{A}\left[\tilde{A}_{1} \rightarrow X\right]=O_{A A} \mathcal{A}\left[A_{1} \rightarrow X\right]+O_{A 3} \mathcal{A}\left[\pi_{3} \rightarrow X\right]+O_{A \eta} \mathcal{A}[\eta \rightarrow X]+O_{A \eta^{\prime}} \mathcal{A}\left[\eta^{\prime} \rightarrow X\right]
$$

where all the amplitudes on the right-hand side should be worked out for the $\tilde{A}_{1}$ kinematics, though.

\footnotetext{
${ }^{1}$ We employ $3,8,9, A$ indices to refer to the $\pi_{3}, \pi_{8}, \pi_{9}, A_{1}$ components; similarly, indices $\eta$ and $\eta^{\prime}$ correspond to a rotation of angle $\theta_{\eta}$ with respect to the $\pi_{8}$ and $\pi_{9}$ components.
} 


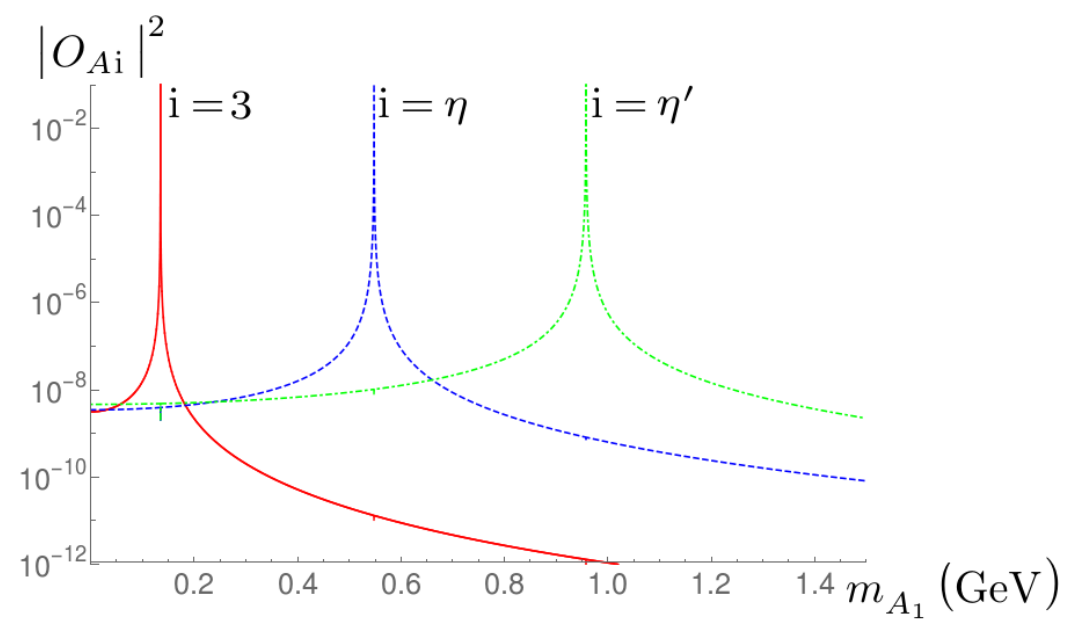

Figure 1. $A_{1}$-meson mixing for $P_{11}=0.03, \tan \beta=10$. The full red line corresponds to $\left|O_{A 3}\right|^{2}$, the dashed blue line, to $\left|O_{A \eta}\right|^{2}$ and the dot-dashed green line, to $\left|O_{A \eta^{\prime}}\right|^{2}$, i.e. the $A_{1}$-pion, $A_{1}-\eta$ and $A_{1}-\eta^{\prime}$ mixing respectively.

\subsection{Photonic decay}

The diphoton decay is one of the channels where the mixing with the mesons has the most dramatic effects for the CP-odd Higgs, due to the large, anomaly-driven diphoton decays of the mesons. The amplitudes can be worked out from eqs. (2.1) and (2.10) - for photons with momenta $p_{1}$ and $p_{2}$ and polarizations $\varepsilon\left(p_{1}\right)$ and $\varepsilon\left(p_{2}\right)$ :

$$
\begin{aligned}
\mathcal{A}\left[A_{1} \rightarrow \gamma \gamma\right] & =\frac{\alpha}{4 \pi}\left(C_{\gamma}+\delta C_{\gamma}^{e, \mu}\right) \varepsilon^{\mu \nu \rho \sigma}\left[p_{1 \mu} \varepsilon_{\nu}\left(p_{1}\right)-p_{1 \nu} \varepsilon_{\mu}\left(p_{1}\right)\right]\left[p_{2 \rho} \varepsilon_{\sigma}\left(p_{2}\right)-p_{2 \sigma} \varepsilon_{\rho}\left(p_{2}\right)\right] \\
\delta C_{\gamma}^{e, \mu} & =-\frac{P_{11}}{2 \sqrt{2} v} Q_{e}^{2} \tan \beta\left\{\mathcal{F}\left(\frac{m_{e}^{2}}{m_{A_{1}}^{2}}\right)+\mathcal{F}\left(\frac{m_{\mu}^{2}}{m_{A_{1}}^{2}}\right)\right\} \\
\mathcal{A}[\Pi \rightarrow \gamma \gamma] & =\frac{\alpha}{4 \pi} C_{\gamma}[\Pi] \varepsilon^{\mu \nu \rho \sigma}\left[p_{1 \mu} \varepsilon_{\nu}\left(p_{1}\right)-p_{1 \nu} \varepsilon_{\mu}\left(p_{1}\right)\right]\left[p_{2 \rho} \varepsilon_{\sigma}\left(p_{2}\right)-p_{2 \sigma} \varepsilon_{\rho}\left(p_{2}\right)\right] ; \Pi=\pi_{3}, \eta, \eta^{\prime} \\
C_{\gamma}\left[\pi_{3}\right] & =\frac{\sqrt{2} N_{c}}{f_{\pi}} \operatorname{Tr}\left[\lambda^{3} Q_{q}^{2}\right] ; \quad C_{\gamma}[\eta]=\frac{\sqrt{2} N_{c}}{f_{\pi}}\left(\cos \theta_{\eta} \operatorname{Tr}\left[\lambda^{8} Q_{q}^{2}\right]-\sin \theta_{\eta} \operatorname{Tr}\left[\lambda^{9} Q_{q}^{2}\right]\right) ; \\
C_{\gamma}\left[\eta^{\prime}\right] & =\frac{\sqrt{2} N_{c}}{f_{\pi}}\left(\sin \theta_{\eta} \operatorname{Tr}\left[\lambda^{8} Q_{q}^{2}\right]+\cos \theta_{\eta} \operatorname{Tr}\left[\lambda^{9} Q_{q}^{2}\right]\right)
\end{aligned}
$$

At leading order, $C_{\gamma}\left[\pi_{3}\right]=-\frac{1}{f_{\pi}}, C_{\gamma}[\eta]=-\frac{1}{\sqrt{3} f_{\pi}}\left(\cos \theta_{\eta}-2 \sqrt{2} \sin \theta_{\eta}\right)$ and $C_{\gamma}\left[\eta^{\prime}\right]=$ $-\frac{1}{\sqrt{3} f_{\pi}}\left(\sin \theta_{\eta}+2 \sqrt{2} \cos \theta_{\eta}\right)$. However, we may exploit the experimental measurements of the $\pi_{3}, \eta$ and $\eta^{\prime}$ diphoton widths [85] to derive more realistic (though close) estimates: $C_{\gamma}\left[\pi_{3}\right] \simeq-10.75 \mathrm{GeV}^{-1}, C_{\gamma}[\eta] \simeq-10.8 \mathrm{GeV}^{-1}$ and $C_{\gamma}\left[\eta^{\prime}\right] \simeq-13.6 \mathrm{GeV}^{-1}$. While we regard this choice as an educated guess resumming higher-order effects, it could be objected that, in so mixing orders, cancellations such as those appearing in the $K_{L}$ diphoton decay amplitude [86] are spoilt. Yet, the properties of the CP-odd Higgs - its mass or its $\tan \beta$-dependent couplings - are not so strictly determined by the $\mathrm{U}(3)_{A}$ symmetry as their $K_{L}$ equivalent, so that we do not expect comparable order-by-order cancellations. Nevertheless, we will compare our result to the case where strict $\mathrm{U}(3)_{A}$ conditions are 
enforced, which translates into a larger value of $\left|\theta_{\eta}\right|$ together with the use of leading-order $C_{\gamma}\left[\pi_{3}, \eta, \eta^{\prime}\right]$ as explicited above. The diphoton width then reads:

$$
\Gamma\left[\tilde{A}_{1} \rightarrow \gamma \gamma\right]=\frac{\alpha^{2} m_{A_{1}}^{3}}{64 \pi^{3}}\left|O_{A A}\left(C_{\gamma}+\delta C_{\gamma}^{e, \mu}\right)+O_{A 3} C_{\gamma}\left[\pi_{3}\right]+O_{A \eta} C_{\gamma}[\eta]+O_{A \eta^{\prime}} C_{\gamma}\left[\eta^{\prime}\right]\right|^{2}
$$

We show $\Gamma\left[\tilde{A}_{1} \rightarrow \gamma \gamma\right]$ in figure 2 for $^{2} P_{11}=0.03, \tan \beta=10$; the result also depends on the chargino contribution to $C_{\gamma}$ : we have employed $M_{2}=\mu_{\text {eff }}=500 \mathrm{GeV}, \lambda=0.3$. The decay width of eq. (3.6) corresponds to the (full) red line. The (dashed) blue curve would correspond to a pure partonic width, neglecting the mixing with the mesons and including a partonic strange quark with mass $95 \mathrm{MeV}$ in $C_{\gamma}$. For the (dot-dashed) green curve, we have neglected the partonic contribution $\left(C_{\gamma}+\delta C_{\gamma}^{e, \mu}\right)$ and assumed that the width would be purely originating in the meson-mixing. Expectedly, this mixing approximation provides a qualitatively good agreement with the full result of eq. (3.6) when $m_{A_{1}}$ is close to a meson mass. On the other hand, the partonic description captures the main effects far from the mixing regime. But it generically falls orders of magnitude away in the vicinity of meson masses. Despite a tiny mixing, we observe that the impact of mesons on the diphoton width extends far beyond the immediate vicinity of the meson masses: this is due to the large mesonic decay widths to photon pairs and the suppressed genuine $A_{1}$ width for a mostly-singlet state. We note that destructive interferences develop among the various amplitudes and, in particular, a local cancellation takes place at $m_{A_{1}} \sim 150 \mathrm{MeV}$. At $m_{A_{1}} \sim 1.5 \mathrm{GeV}$, the impact of the mixing with the $\eta^{\prime}$ wanes, leaving the partonic description in a satisfactory posture again. Finally, the orange dotted curve corresponds to the case where strict $\mathrm{U}(3)_{A}$ relations have been enforced, at the level of the mixing among pions $\left(\theta_{\eta}\right)$, between pions and the Higgs state or in the expression of the pionphoton couplings. We observe minor differences, in particular a small suppression of the $\eta$-driven mixing contribution, but the Higgs width essentially retains the qualitative trends discussed before. The deviation between the red and orange curves is understood as a higher-order effect and, as such, part of the error estimate. While we stick to the more phenomenological approach below, it is difficult to choose which of the two approaches is actually more reliable at this level.

\subsection{Leptonic decays}

The situation is reversed for the leptonic decays: the corresponding branching ratios for the mesons are typically tiny, while the leptonic decays of the Higgs state, $\tan \beta$-enhanced, would naively count among the dominant channels at low-mass. Consequently, the muonic and electronic widths of the $\tilde{A}_{1}$ state are well approximated by their pure-Higgs analogues. On the contrary, the mixing with the mesons generates an additional leptonic width for the $\pi_{3}, \eta$ and $\eta^{\prime}$, which represents the main limiting factor on this mixing effect.

\footnotetext{
${ }^{2}$ Most of the amplitudes (or mixing elements) involving $A_{1}$ depend linearly on $P_{11}$. The only exception is $C_{\gamma}$ where a direct coupling of the higgsinos to the singlet component of $A_{1}$ intervenes. Although we confine to the case $P_{11}=0.03$ in numerical applications, other choices could thus be easily reconstructed via a rescaling of the widths by $\sim\left(\frac{P_{11}}{0.03}\right)^{2}$.
} 


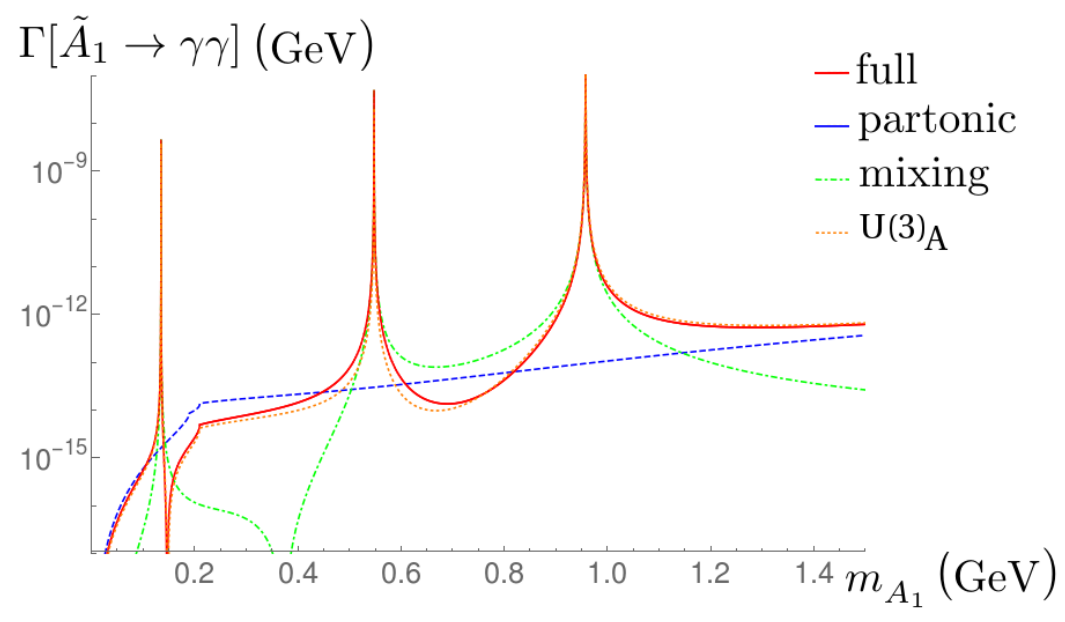

Figure 2. Diphoton width of the mostly- $A_{1}$ state. $P_{11}=0.03, \tan \beta=10, M_{2}=\mu_{\text {eff }}=500 \mathrm{GeV}$, $\lambda=0.3$. In (dashed) blue is the partonic width (including a partonic strange quark with mass $0.095 \mathrm{GeV}$ ). The (dotdashed) green curve corresponds to the width mediated by the meson mixing (i.e. neglecting $C_{\gamma}$ ). The full result of eq. (3.6), employing phenomenological estimates of the pionphoton couplings, is in red (full line). For the orange dotted curve, we have imposed strict $\mathrm{U}(3)_{A}$ relations at all levels $\left(\eta-\eta^{\prime}\right.$-mixing, $A_{1}$-pion mixings, pion-photon couplings).

We may express the leptonic $(l=e, \mu)$ decay width of a pseudoscalar state $P=$ $A_{1}, \pi_{3}, \eta, \eta^{\prime}$ as:

$$
\Gamma\left[P \rightarrow l^{+} l^{-}\right]=\frac{\left|Y_{P}^{l l}\right|^{2}}{8 \pi} m_{P} \sqrt{1-\frac{4 m_{l}^{2}}{m_{P}^{2}}} ; \quad Y_{A_{1}}^{l l}=\frac{m_{l}}{\sqrt{2} v} P_{11} \tan \beta
$$

A few effective mesonic couplings can be estimated numerically from the experimental measurements [85]: $Y_{\pi_{3}}^{e e} \simeq 3 \cdot 10^{-7} ; Y_{\eta}^{\mu \mu} \simeq 2 \cdot 10^{-5}$. However, only upper limits are available for the $e^{+} e^{-}$decays of the $\eta$ and $\eta^{\prime}$ and the $\mu^{+} \mu^{-}$decay of $\eta^{\prime}$ is uncharted. We thus neglect such missing input.

We show the leptonic decay widths of the mixed-state $\tilde{A}_{1}$ in figure 3 , for $P_{11}=0.03$, $\tan \beta=10$. We observe that, except for the immediate vicinity of the meson masses, the leptonic decays are essentially determined by the pure-Higgs widths.

As we mentioned above, the mixing induces an additional leptonic width for the mesons. This is actually the main impact of the mixing from the perspective of the mesons. As the measured leptonic decays are typically small, we may place some limits on this scenario. For instance, still in the case $P_{11}=0.03, \tan \beta=10, \Gamma\left[\tilde{\pi}^{0} \rightarrow e^{+} e^{-}\right]$would fall $\sim 20 \%$ beyond its experimental central value when $m_{A_{1}}$ is within $\sim 0.15 \mathrm{MeV}$ of $m_{\pi}$. Similarly, in a $\sim 3 \mathrm{MeV}$-wide mass-window centered on $m_{\eta}, \Gamma\left[\tilde{\eta} \rightarrow \mu^{+} \mu^{-}\right]$is $\sim 30 \%$ off. On the other hand, the limits on $\Gamma\left[\tilde{\eta} \rightarrow e^{+} e^{-}\right]$and $\Gamma\left[\tilde{\eta}^{\prime} \rightarrow e^{+} e^{-}\right]$are well satisfied. We note, however, that such limits only apply if one assumes that the measured $\Gamma\left[\tilde{\pi}^{0} \rightarrow e^{+} e^{-}\right]$or $\Gamma\left[\tilde{\eta} \rightarrow \mu^{+} \mu^{-}\right]$ are exactly explained by the SM. When the SM is off, the mixing effect could well improve the agreement with the measured value. Such a point was actually discussed in [58] in the case of $\Gamma\left[\tilde{\pi}^{0} \rightarrow e^{+} e^{-}\right]$, as one may choose to see some tension between the experimental measurement [87] and the theoretical evaluation [88]. Thus, exclusion of mass-values for 


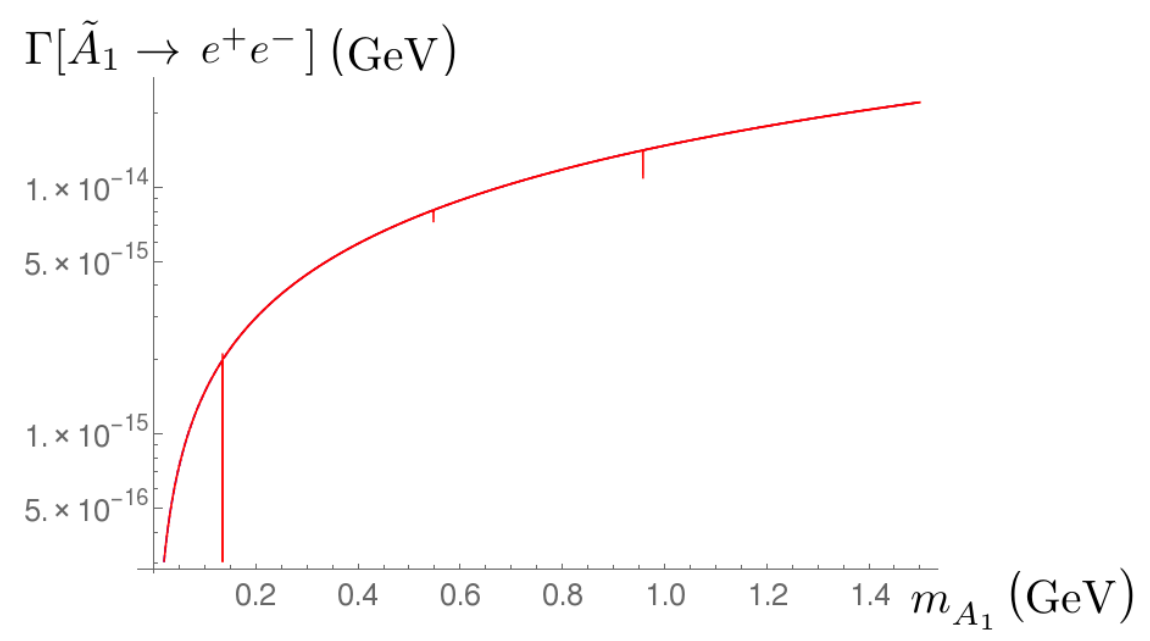

$\Gamma\left[\tilde{A}_{1} \rightarrow \mu^{+} \mu^{-}\right](\mathrm{GeV})$

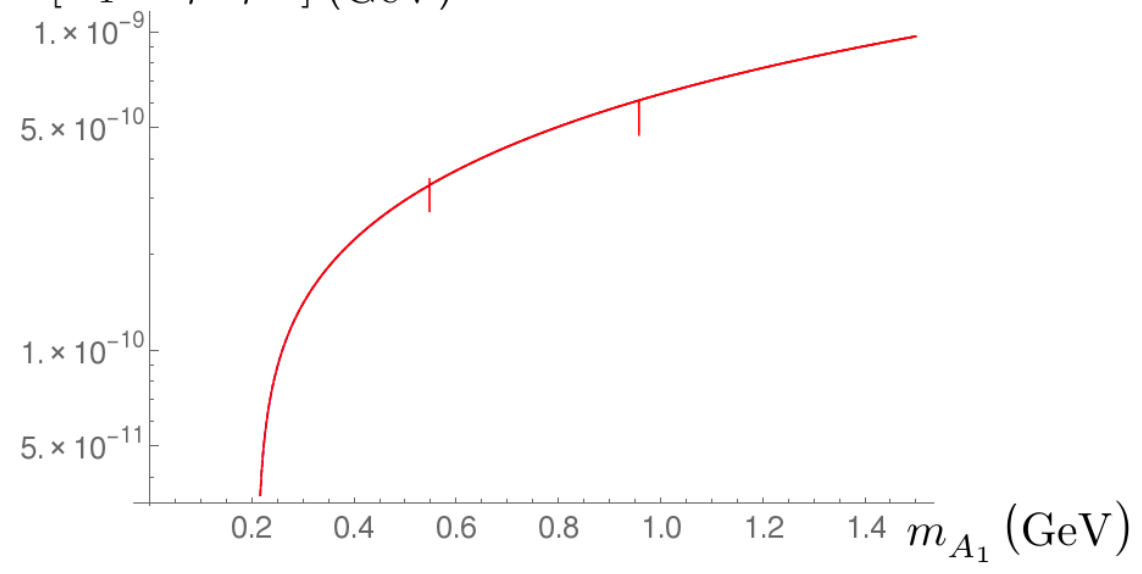

Figure 3. Leptonic width of the $\tilde{A}_{1}$ state. $P_{11}=0.03, \tan \beta=10$. The full result (including mixing) is shown as a (full) red line. It essentially covers the (dashed) blue curve, corresponding to a pure-Higgs decay.

$A_{1}$ close to a meson mass strongly depends on the assumptions and a detailed analysis would prove necessary.

\subsection{Tri-meson decays}

The decay width of a pseudoscalar $P$ to a tri-mesonic final state $\Pi_{i} \Pi_{j} \Pi_{k}$ may be written as:

$$
\begin{aligned}
\Gamma\left[P \rightarrow \Pi_{i} \Pi_{j} \Pi_{k}\right]= & \frac{1}{256 S_{i j k} \pi^{3} m_{P}} \int_{\left(m_{j}+m_{k}\right)^{2}}^{\left(m_{P}-m_{i}\right)^{2}} d s\left|\mathcal{A}_{P}^{i j k}\right|^{2} \sqrt{1-\frac{2\left(m_{j}^{2}+m_{k}^{2}\right)}{s}+\frac{\left(m_{j}^{2}-m_{k}^{2}\right)^{2}}{s^{2}}} \\
& \times \sqrt{\left(1+\frac{s-m_{i}^{2}}{m_{P}^{2}}\right)^{2}-\frac{4 s}{m_{P}^{2}}}
\end{aligned}
$$

where $m_{P, i, j, k}$ stand for the masses of $P, \Pi_{i}, \Pi_{j}, \Pi_{k}$ and $S_{i j k}$ is a symmetry factor: 1 , 2 or 3 ! depending on the number of identical particles in the final state. The transition 
amplitude $\mathcal{A}_{P}^{i j k}$ should be determined from the chiral lagrangian. Expliciting the quartic Higgs-meson couplings in eq. (2.10) provides us with:

$$
\begin{aligned}
& \tilde{\mathcal{L}}_{\chi} \ni-\frac{P_{11} A_{1}}{12 \sqrt{2} v f_{\pi}} \cdot\left(\pi_{3}^{2}+2 \pi^{+} \pi^{-}\right)\left\{\left[m_{\pi}^{2}\left(\tan ^{-1} \beta-\tan \beta\right)+\delta\left(\tan ^{-1} \beta+\tan \beta\right)\right] \pi_{3}\right. \\
& \left.+\sqrt{3}\left[m_{\pi}^{2}\left(\tan ^{-1} \beta+\tan \beta\right)+\delta\left(\tan ^{-1} \beta-\tan \beta\right)\right]\left[\left(\cos \theta_{\eta}-\sqrt{2} \sin \theta_{\eta}\right) \eta+\left(\sin \theta_{\eta}+\sqrt{2} \cos \theta_{\eta}\right) \eta^{\prime}\right]\right\} \\
& -\frac{P_{11} A_{1}}{6 \sqrt{2} v f_{\pi}} \cdot \pi_{3}\left\{\left[m_{K^{ \pm}}^{2}\left(2 \tan ^{-1} \beta+\tan \beta\right)+\left(m_{\pi}^{2}-m_{K^{0}}^{2}\right)\left(2 \tan ^{-1} \beta-\tan \beta\right)\right] K^{+} K^{-}\right. \\
& \left.+\left[m_{K^{ \pm}}^{2}-m_{\pi}^{2}-3 m_{K^{0}}^{2}\right] \tan \beta K^{0} \bar{K}^{0}\right\} \\
& -\frac{P_{11} A_{1}}{6 v f_{\pi}} \cdot\left(K^{0} K^{-} \pi^{+}+\bar{K}^{0} K^{+} \pi^{-}\right)\left[\left(m_{K^{ \pm}}^{2}+m_{\pi}^{2}\right) \tan ^{-1} \beta-m_{K^{0}}^{2}\left(\tan ^{-1} \beta-2 \tan \beta\right)\right] \\
& -\frac{P_{11} A_{1}}{12 \sqrt{2} v f_{\pi}}\left[m_{\pi}^{2}\left(\tan ^{-1} \beta-\tan \beta\right)+\delta\left(\tan ^{-1} \beta+\tan \beta\right)\right] \pi_{3} \\
& \times\left[\left(\cos \theta_{\eta}-\sqrt{2} \sin \theta_{\eta}\right) \eta+\left(\sin \theta_{\eta}+\sqrt{2} \cos \theta_{\eta}\right) \eta^{\prime}\right]^{2} \\
& \tilde{\mathcal{L}}_{\chi} \ni \frac{m_{\pi}^{2}}{24 f_{\pi}^{2}}\left(\pi_{3}^{4}+4 \pi_{3}^{2} \pi^{+} \pi^{-}\right) \\
& +\frac{\delta}{6 \sqrt{3} f_{\pi}^{2}}\left[\left(\cos \theta_{\eta}-\sqrt{2} \sin \theta_{\eta}\right) \eta+\left(\sin \theta_{\eta}+\sqrt{2} \cos \theta_{\eta}\right) \eta^{\prime}\right] \pi_{3}\left(\pi_{3} \pi_{3}+2 \pi^{+} \pi^{-}\right) \\
& +\frac{m_{\pi}^{2}}{12 f_{\pi}^{2}}\left[\left(\cos \theta_{\eta}-\sqrt{2} \sin \theta_{\eta}\right) \eta+\left(\sin \theta_{\eta}+\sqrt{2} \cos \theta_{\eta}\right) \eta^{\prime}\right]^{2}\left(\pi_{3} \pi_{3}+2 \pi^{+} \pi^{-}\right) \\
& +\frac{1}{12 f_{\pi}^{2}}\left\{\left(2 m_{K^{ \pm}}^{2}-m_{K^{0}}^{2}+m_{\pi}^{2}\right) \pi_{3}^{2} K^{+} K^{-}+\left(2 m_{K^{0}}^{2}-m_{K^{ \pm}}^{2}+m_{\pi}^{2}\right) \pi_{3}^{2} K^{0} \bar{K}^{0}\right. \\
& \left.+\sqrt{2} \delta \pi_{3}\left(\pi^{+} K^{-} K^{0}+\pi^{-} K^{+} \bar{K}^{0}\right)\right\} \\
& +\frac{1}{6 \sqrt{3} f_{\pi}^{2}}\left\{\left[-3 \sqrt{2} m_{K^{ \pm}}^{2} \sin \theta_{\eta}+\left(m_{\pi}^{2}-m_{K^{0}}^{2}\right)\left(\cos \theta_{\eta}-\sqrt{2} \sin \theta_{\eta}\right)\right] \eta\right. \\
& \left.+\left[3 \sqrt{2} m_{K^{ \pm}}^{2} \cos \theta_{\eta}+\left(m_{\pi}^{2}-m_{K^{0}}^{2}\right)\left(\sin \theta_{\eta}+\sqrt{2} \cos \theta_{\eta}\right)\right] \eta^{\prime}\right\} \pi_{3} K^{+} K^{-} \\
& +\frac{1}{6 \sqrt{3} f_{\pi}^{2}}\left\{\left[3 \sqrt{2} m_{K^{0}}^{2} \sin \theta_{\eta}+\left(m_{K^{ \pm}}^{2}-m_{\pi}^{2}\right)\left(\cos \theta_{\eta}-\sqrt{2} \sin \theta_{\eta}\right)\right] \eta\right. \\
& \left.+\left[-3 \sqrt{2} m_{K^{0}}^{2} \cos \theta_{\eta}-\left(m_{K^{ \pm}}^{2}-m_{\pi}^{2}\right)\left(\sin \theta_{\eta}+\sqrt{2} \cos \theta_{\eta}\right)\right] \eta^{\prime}\right\} \pi_{3} K^{0} \bar{K}^{0} \\
& +\frac{1}{6 \sqrt{6} f_{\pi}^{2}}\left\{\left[\left(2 m_{\pi}^{2}-m_{K^{ \pm}}^{2}-m_{K^{0}}^{2}\right) \cos \theta_{\eta}-2 \sqrt{2}\left(m_{K^{0}}^{2}+m_{K^{ \pm}}^{2}+m_{\pi}^{2}\right) \sin \theta_{\eta}\right] \eta\right. \\
& \left.+\left[\left(2 m_{\pi}^{2}-m_{K^{ \pm}}^{2}-m_{K^{0}}^{2}\right) \sin \theta_{\eta}+2 \sqrt{2}\left(m_{K^{0}}^{2}+m_{K^{ \pm}}^{2}+m_{\pi}^{2}\right) \cos \theta_{\eta}\right] \eta^{\prime}\right\}\left(\pi^{+} K^{-} K^{0}+\pi^{-} K^{+} \bar{K}^{0}\right) \\
& +\frac{\delta}{18 \sqrt{3} f_{\pi}^{2}} \pi_{3}\left\{\left(\cos \theta_{\eta}-\sqrt{2} \sin \theta_{\eta}\right) \eta+\left(\sin \theta_{\eta}+\sqrt{2} \cos \theta_{\eta}\right) \eta^{\prime}\right\}^{3}
\end{aligned}
$$

Starting from $m_{A_{1}} \gtrsim 3 m_{\pi}$, the tri-pion decays of $A_{1}$ become kinematically accessible. The chiral lagrangian contains $A_{1}$-pion couplings explicitly, $\propto \frac{m_{\pi}^{2}}{v f_{\pi}} P_{11}\left(\tan ^{-1} \beta-\tan \beta\right)$. The $\eta$ and $\eta^{\prime}$ couplings to three pions are isospin-violating $(\propto \delta)$. Diagrams involving the $\eta / \eta^{\prime}-$ $\pi_{3}$ mixing thus contribute at the same order in $\delta[89,90]$. The basic evaluation employing $\delta \simeq m_{K^{ \pm}}^{2}-m_{K^{0}}^{2}-m_{\pi^{ \pm}}^{2}+m_{\pi}^{2}$ is known to provide a substantially smaller contribution to $\Gamma[\eta \rightarrow 3 \pi]$ than experiments indicate [91]. In practice we thus rescale the corresponding couplings by comparison with the experimental widths $\Gamma[\eta \rightarrow 3 \pi]$ and $\Gamma\left[\eta^{\prime} \rightarrow 3 \pi\right]$ directly. 

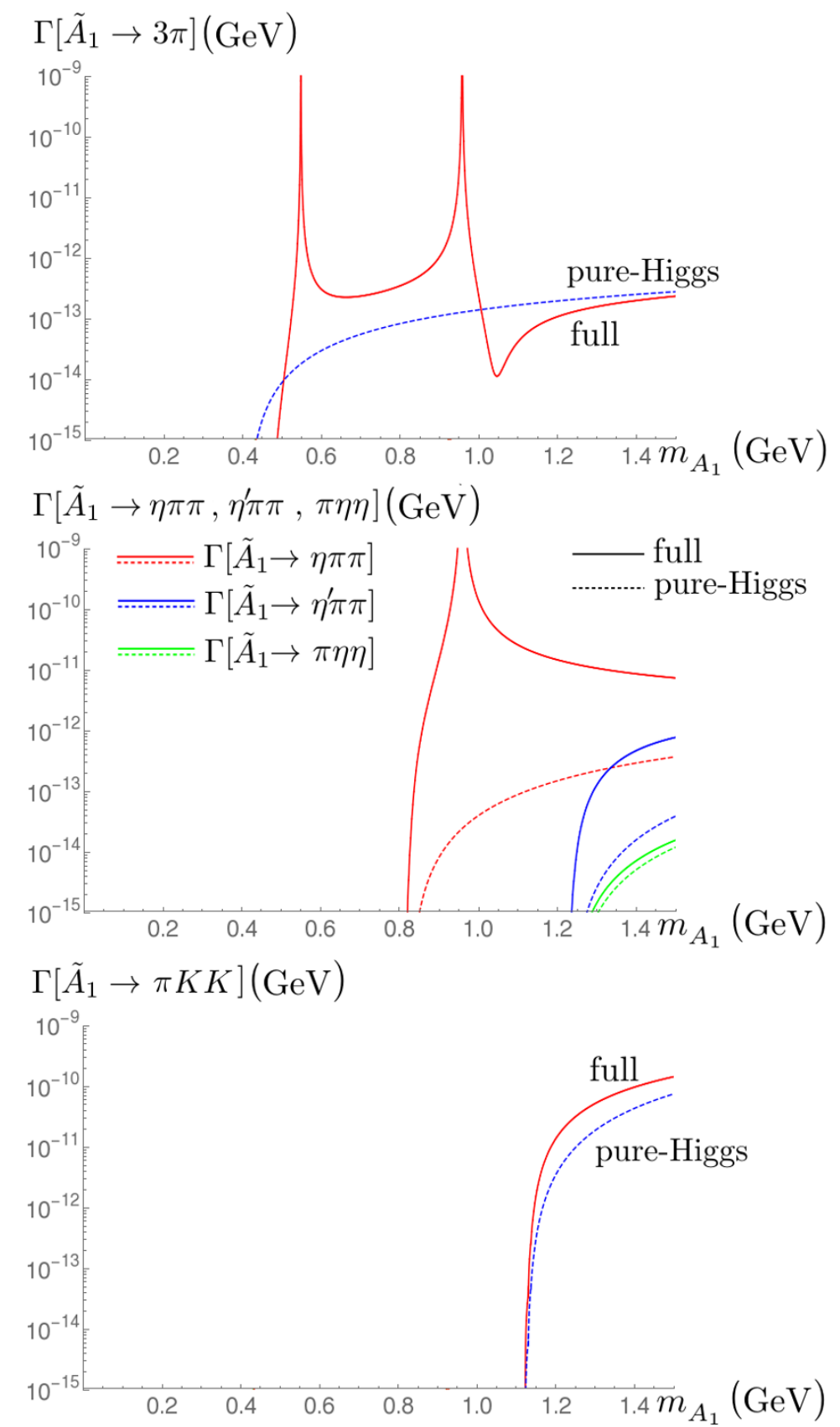

Figure 4. Hadronic decay widths to for $P_{11}=0.03, \tan \beta=10$. The plot above corresponds to the $\tilde{A}_{1} \rightarrow 3 \pi$ channel: the full result (including mixing) is shown as a (full) red line while the (dashed) blue curve corresponds to a pure-Higgs decay. The plot in the middle shows the $\tilde{A}_{1} \rightarrow \eta \pi \pi$ (red curves: full $\rightarrow$ with mixing; dashed $\rightarrow$ without mixing), $\tilde{A}_{1} \rightarrow \eta^{\prime} \pi \pi$ (blue curves) and $\tilde{A}_{1} \rightarrow \pi \eta \eta$ (green curves) widths. The plot on the bottom corresponds to the $\tilde{A}_{1} \rightarrow \pi K K$ channels (red full curve: with mixing; blue dashed cure: without). 
We show the corresponding estimate of $\Gamma\left[\tilde{A}_{1} \rightarrow 3 \pi\right]$ (summing over neutral and charged pionic final states) for $P_{11}=0.03, \tan \beta=10$ in the upper part of figure 4 . The full result (red full curve) differs again sizably from the pure-Higgs width (blue dashed line) due to the large impact of the $\eta$ and $\eta^{\prime}$ resonances.

The $\eta \pi \pi$ channels open up at $m_{A_{1}} \gtrsim 820 \mathrm{MeV}$. In the case of the $\eta^{\prime}$, they represent one of the main final states - the $\eta^{\prime} \eta \pi \pi$ coupling is isospin-conserving $\propto \frac{m_{\pi}^{2}}{f_{\pi}^{2}}$, though the decay is phase-space suppressed. Again, we rescale the couplings of eq. (3.10) in order to account for the experimental $\Gamma\left[\eta^{\prime} \rightarrow \eta \pi \pi\right]$. Similarly, we include the $\eta^{\prime} \pi \pi$ and $\pi_{3} \eta \eta$ channels at $m_{A_{1}} \gtrsim 1.23 \mathrm{GeV}$. It is remarkable that in none of these decays the coupling $\propto \frac{B m_{s}}{f_{\pi}}$ intervenes. The results are displayed in the plot in the middle of figure $4: \tilde{A}_{1} \rightarrow \eta \pi \pi$ (red lines) expectedly proves the most relevant of these channels. The very large $\eta^{\prime} \rightarrow \eta \pi \pi$ decay induces a sizable deviation of the $\tilde{A}_{1}$ decay (full curve) as compared to the pure-Higgs amplitude (dashed curve) and this effect is still partially affecting the $\tilde{A}_{1} \rightarrow \eta \pi \pi$ width at $m_{A_{1}} \sim 1.5 \mathrm{GeV}$.

Beyond $m_{A_{1}} \gtrsim 1.12 \mathrm{GeV}$, the $\pi K K$ channels are accessible in their turn. They are the first decays to employ the coupling $\propto \frac{B m_{s}}{f_{\pi}}$, meaning that the impact of the strange quark on the $\tilde{A}_{1}$ width is kinematically delayed till this quite-high threshold. We cannot use experimental data to evaluate the $\pi_{3}, \eta$ or $\eta^{\prime}$ couplings to the corresponding final states in a phenomenologically more efficient way than employing eq. (3.10). However, we note that the kinematically relevant region is already far above the masses of the pseudoscalar mesons, so that the mixing effect should be subdominant. Our result (summing over the $\pi_{3} K^{+} K^{-}, \pi_{3} K^{0} \bar{K}^{0}, \pi^{+} K^{-} K^{0}$ and $\pi^{-} K^{+} \bar{K}^{0}$ final states) is displayed in the lower plot of figure 4 . These kaonic widths are typically one to two orders of magnitude larger than the pionic decays, due to the larger coupling. The mixing effect appears to affect these decay channels in a subdominant way, although a small excess is still visible at $m_{A_{1}} \sim 1.5 \mathrm{GeV}$.

Other tri-meson final states include $\pi_{3} \eta \eta^{\prime}, \pi_{3} \eta^{\prime} \eta^{\prime}, 3 \eta, \eta^{\prime} \eta \eta, \eta \eta^{\prime} \eta^{\prime}, 3 \eta^{\prime}, \eta K^{+} K^{-}$, $\eta^{\prime} K^{+} K^{-}, \eta K^{0} \bar{K}^{0}$ and $\eta^{\prime} K^{0} \bar{K}^{0}$. They intervene only beyond $m_{A_{1}}>1.5 \mathrm{GeV}$ so we leave the corresponding description to the following section.

\subsection{Radiative hadronic decays}

The decays $\eta \rightarrow \gamma \pi^{+} \pi^{-}$and $\eta^{\prime}(\rightarrow \gamma \rho, \omega) \rightarrow \gamma \pi^{+} \pi^{-}$show the relevance of radiative decay modes for light pseudoscalar states. Such decays are entirely specified by the anomaly and (in the case of pseudoscalar mesons) they are well described in a Vector Dominance approach — see e.g. [91]. For the Higgs pseudoscalar, we confine to the leading-order, in which the radiative hadronic decays result from the mixing effect with the pseudoscalar mesons.

We follow the description of [91] for the $\eta / \eta^{\prime} \rightarrow \gamma \pi^{+} \pi^{-}$decay and correct the small mismatch with the experimental widths by a rescaling factor. Then we display the decay width acquired by $\tilde{A}_{1}$ via mixing in figure 5 . Expectedly, the effect is largest close to the $\eta$ and $\eta^{\prime}$ masses. Yet, the tail at $m_{A_{1}} \simeq 1.5 \mathrm{GeV}$ competes in magnitude with the pionic decay widths. We may wonder whether large $\gamma K K$ decays could not develop via the mediation of the $\phi$. We will assume that it is not the case, first because the $\phi$ is much narrower than the $\rho$, second because we believe that these radiative decays remain subdominant as compared to the $\pi K K$ channels. 


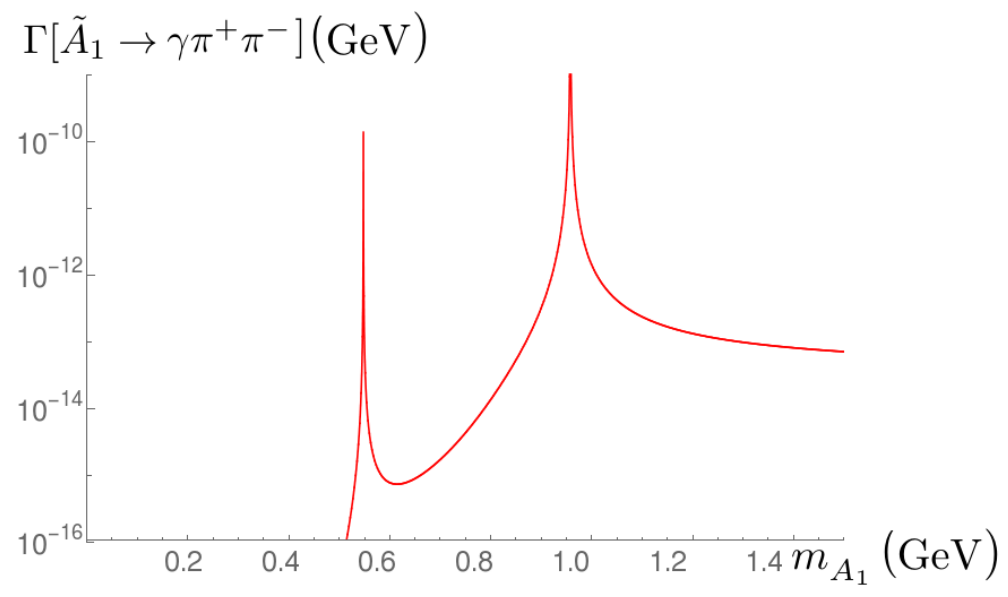

Figure 5. $\tilde{A}_{1} \rightarrow \gamma \pi^{+} \pi^{-}$width for $P_{11}=0.03, \tan \beta=10$. In our approach, this decay is only mediated by the mixing with the pseudoscalar mesons.

\subsection{Decays in the chiral limit: summary}

At this level, we believe to have considered the major possible decay channels to SM particles for the CP-odd Higgs in the chiral limit. These are summarized in figure 6: as was already pointed out by e.g. [55], the leptonic widths (blue dashed curve) dominate most of the low-mass region. One then naively expects severe constraints from e.g. flavour observables, where, however, the interplay of supersymmetric contributions in the flavourchanging $A_{1}$ couplings should be considered carefully: such a discussion goes beyond the aims of the present paper. However, close to the mass of the $\pi_{3}, \eta$ or $\eta^{\prime}$, the Higgs pseudoscalar may have enhanced decays to a photon pair (red full curve) or to hadrons (green dot-dashed curve) and these final states may compete with the dimuon channel. At $m_{A_{1}} \simeq 1.5 \mathrm{GeV}$, the hadronic decays still represent only $\sim 10 \%$ of the SM width of $\tilde{A}_{1}$ (for the particular values $P_{11}=0.03, \tan \beta=10$ ).

As singlino Dark Matter is a motivation for the light $A_{1}$ scenario, we note that an invisible decay into singlinos $\left(\tilde{\chi}_{s}^{0}\right)$ could be relevant if the $A_{1}$ mass is above threshold. We expect this channel to be dominated by the singlet-singlino interactions. At leading order:

$$
\Gamma\left[\tilde{A}_{1} \rightarrow \tilde{\chi}_{s}^{0} \tilde{\chi}_{s}^{0}\right]=\frac{\kappa^{2}\left(1-P_{11}^{2}\right)}{8 \pi} m_{A_{1}} \sqrt{1-\frac{4 m_{\tilde{\chi}_{s}^{0}}}{m_{A_{1}}}}
$$

For the thermal annihilation of singlinos in the early universe to be efficient enough so that we avoid excessive relics - , the corresponding cross-section must be enhanced by a resonant $A_{1}$, i.e. $m_{A_{1}}$ should be close to the energy of the singlino pair maximizing the Boltzmann distribution at the freeze-out temperature, i.e $m_{A_{1}} \simeq 2 m_{\tilde{\chi}_{s}^{0}}$. Then, the phase-space of the decay is suppressed. Moreover, observing that the singlino mass in the $Z_{3}$-conserving NMSSM is given by $m_{\tilde{\chi}_{s}^{0}} \simeq 2 \kappa s$, it seems natural to turn to the PecceiQuinn limit $(\kappa \rightarrow 0)$ to ensure both a light $A_{1}$ and a light singlino. Eq. (3.11) is further suppressed in this limit. However, in view of $m_{A_{1}} \simeq 2 m_{\tilde{\chi}_{s}^{0}}$, threshold corrections would also be relevant. We will not enter into more details here as we are chiefly interested in the SM decays of the CP-odd Higgs. We remind that sizable invisible $A_{1}$ decays could 


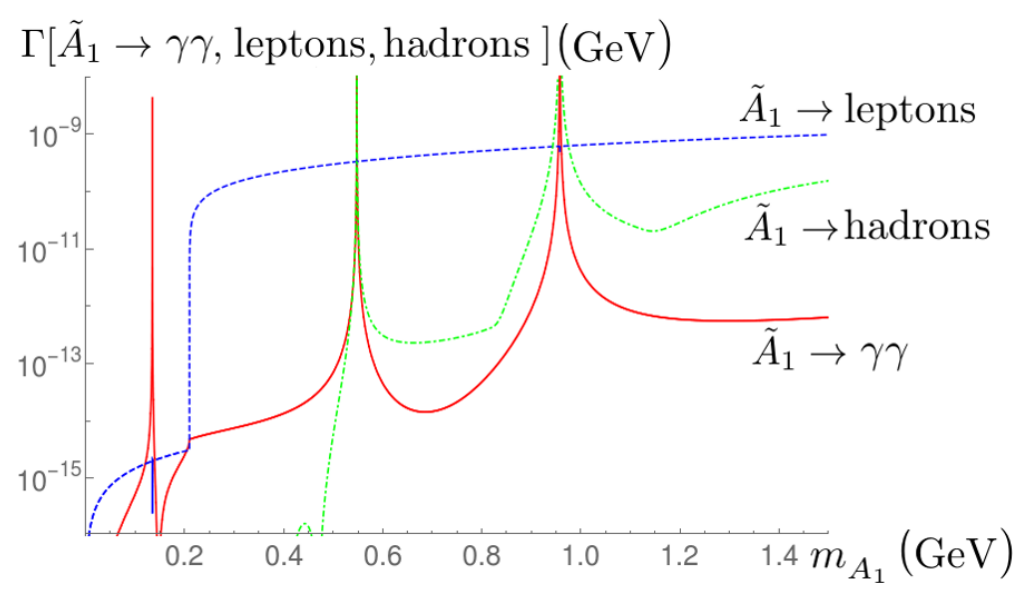

Figure 6. Summary of the $\tilde{A}_{1}$ decays in the chiral limit for $P_{11}=0.03, \tan \beta=10$ : the full red line corresponds to the diphoton decay, the blue dashed curve, to the leptonic decays, and the green dot-dashed curve sums all hadronic decays.

fall under direct limits from $K$ or $B$ decays, since hadronic processes may be converted to hadronic+invisible, which receive tight phenomenological constraints. Similarly to the case of large leptonic decays, the impact of such limits on the details of the supersymmetric spectrum should be studied carefully, which is beyond the scope of the present paper.

\section{Beyond the chiral limit}

In the previous section, we have seen how the interactions of a light CP-odd Higgs with the strong-interacting sector could be described in a chiral lagrangian. The mixing of $A_{1}$ with the neutral pseudoscalar mesons appeared as an important ingredient, coupling the Higgs pseudoscalar to the chiral anomaly and modifying its hadronic decays. Yet, beyond $m_{A_{1}} \gtrsim 1 \mathrm{GeV}$, one becomes suspicious as to the validity of the chiral description and one prefers to turn to a more partonic approach, the perturbative spectator model [26, 55, 92]. This effective approach essentially adopts a partonic dynamics while keeping the kinematics of the hadrons. In this section, we re-cast the hadronic decays of the Higgs pseudoscalar in terms of this partonic description.

We thus consider the following effective lagrangian for the interaction of $A_{1}$ with the partons:

$$
\mathcal{L}_{\text {spect. }}=\frac{\imath}{\sqrt{2}} A_{1}\left\{\mathcal{Y}_{u}^{A} \bar{u} \gamma_{5} u+\mathcal{Y}_{d}^{A} \bar{d} \gamma_{5} d+\mathcal{Y}_{s}^{A} \bar{s} \gamma_{5} s\right\}
$$

$\mathcal{Y}_{u}^{A}, \mathcal{Y}_{d}^{A}$ and $\mathcal{Y}_{s}^{A}$ are effective Yukawa couplings that should be identified with the chiral couplings in the chiral limit. The partonic amplitudes, stripped of the spinors (since these affect the kinematics), are particularly simple and read:

$$
\begin{aligned}
& \tilde{\mathcal{A}}\left[A_{1} \rightarrow u \bar{u}\right]=\frac{1}{\sqrt{2}} \mathcal{Y}_{u}^{A} \\
& \tilde{\mathcal{A}}\left[A_{1} \rightarrow d \bar{d}\right]=\frac{1}{\sqrt{2}} \mathcal{Y}_{d}^{A} \\
& \tilde{\mathcal{A}}\left[A_{1} \rightarrow s \bar{s}\right]=\frac{1}{\sqrt{2}} \mathcal{Y}_{s}^{A}
\end{aligned}
$$


Our concern now consists in distributing this dynamics among the hadronic channels. We shall assume that these are dominated by the tri-meson final states.

Forgetting momentarily about the mixing effect (i.e. we focus on genuine Higgs amplitudes below), it is useful to notice that the chiral amplitudes of eq. (3.8) satisfy the property:

$$
\begin{aligned}
\sum_{(i, j, k)} \frac{1}{S_{i j k}}\left|\mathcal{A}_{P}^{i j k}\right|^{2}=18\left(\frac{B P_{11}}{6 v f_{\pi}^{2}}\right)^{2}\left[\left(\frac{m_{u}}{\tan \beta}\right)^{2}\right. & +\left(m_{d} \tan \beta\right)^{2}+\left(m_{s} \tan \beta\right)^{2} \\
& \left.+2 m_{u} m_{d}+2 m_{u} m_{s}+2 m_{d} m_{s} \tan ^{2} \beta\right]
\end{aligned}
$$

If we discard the terms of the second line, subleading in $m_{s}$ or in $\tan \beta$ in individual amplitudes, we may identify eq. (4.3) with its partonic analogue $\frac{N_{c}}{2}\left[\left(\mathcal{Y}_{u}^{A}\right)^{2}+\left(\mathcal{Y}_{d}^{A}\right)^{2}+\left(\mathcal{Y}_{s}^{A}\right)^{2}\right]$ and come to the relations:

$$
\mathcal{Y}_{u}^{A} \simeq \frac{B P_{11}}{\sqrt{3} v f_{\pi}^{2}} \frac{m_{u}}{\tan \beta} ; \quad \mathcal{Y}_{d}^{A} \simeq \frac{B P_{11}}{\sqrt{3} v f_{\pi}^{2}} m_{d} \tan \beta ; \quad \mathcal{Y}_{s}^{A} \simeq \frac{B P_{11}}{\sqrt{3} v f_{\pi}^{2}} m_{s} \tan \beta
$$

Eq. (4.3) also hints at how to distribute the partonic amplitude among the 21 tri-meson final states:

- $\tilde{\mathcal{A}}^{2}\left[A_{1} \rightarrow 3 \pi\right]=\frac{5}{144} N_{c}\left[\left(\mathcal{Y}_{u}^{A}\right)^{2}+\left(\mathcal{Y}_{d}^{A}\right)^{2}\right]$

- $\tilde{\mathcal{A}}^{2}\left[A_{1} \rightarrow \eta \pi \pi\right]=\frac{1}{16} N_{c}\left[\left(\mathcal{Y}_{u}^{A}\right)^{2}+\left(\mathcal{Y}_{d}^{A}\right)^{2}\right]\left(\cos \theta_{\eta}-\sqrt{2} \sin \theta_{\eta}\right)^{2}$

- $\tilde{\mathcal{A}}^{2}\left[A_{1} \rightarrow \eta^{\prime} \pi \pi\right]=\frac{1}{16} N_{c}\left[\left(\mathcal{Y}_{u}^{A}\right)^{2}+\left(\mathcal{Y}_{d}^{A}\right)^{2}\right]\left(\sin \theta_{\eta}+\sqrt{2} \cos \theta_{\eta}\right)^{2}$

- $\tilde{\mathcal{A}}^{2}\left[A_{1} \rightarrow \pi \eta \eta\right]=\frac{1}{144} N_{c}\left[\left(\mathcal{Y}_{u}^{A}\right)^{2}+\left(\mathcal{Y}_{d}^{A}\right)^{2}\right]\left(\cos \theta_{\eta}-\sqrt{2} \sin \theta_{\eta}\right)^{4}$

- $\tilde{\mathcal{A}}^{2}\left[A_{1} \rightarrow \pi \eta \eta^{\prime}\right]=\frac{1}{72} N_{c}\left[\left(\mathcal{Y}_{u}^{A}\right)^{2}+\left(\mathcal{Y}_{d}^{A}\right)^{2}\right]\left(\cos \theta_{\eta}-\sqrt{2} \sin \theta_{\eta}\right)^{2}\left(\sin \theta_{\eta}+\sqrt{2} \cos \theta_{\eta}\right)^{2}$;

- $\tilde{\mathcal{A}}^{2}\left[A_{1} \rightarrow \pi \eta^{\prime} \eta^{\prime}\right]=\frac{1}{144} N_{c}\left[\left(\mathcal{Y}_{u}^{A}\right)^{2}+\left(\mathcal{Y}_{d}^{A}\right)^{2}\right]\left(\sin \theta_{\eta}+\sqrt{2} \cos \theta_{\eta}\right)^{4}$

- $\tilde{\mathcal{A}}^{2}\left[A_{1} \rightarrow 3 \eta\right]=\frac{1}{1296} N_{c}\left[\left(\mathcal{Y}_{u}^{A}\right)^{2}+\left(\mathcal{Y}_{d}^{A}\right)^{2}+64\left(\mathcal{Y}_{s}^{A}\right)^{2}\right]$ (in the approximation $\theta_{\eta} \simeq 0$ );

- $\tilde{\mathcal{A}}^{2}\left[A_{1} \rightarrow \eta \eta \eta^{\prime}\right]=\frac{1}{216} N_{c}\left[\left(\mathcal{Y}_{u}^{A}\right)^{2}+\left(\mathcal{Y}_{d}^{A}\right)^{2}+16\left(\mathcal{Y}_{s}^{A}\right)^{2}\right]$ (in the approximation $\theta_{\eta} \simeq 0$ );

- $\tilde{\mathcal{A}}^{2}\left[A_{1} \rightarrow \eta \eta^{\prime} \eta^{\prime}\right]=\frac{1}{108} N_{c}\left[\left(\mathcal{Y}_{u}^{A}\right)^{2}+\left(\mathcal{Y}_{d}^{A}\right)^{2}+4\left(\mathcal{Y}_{s}^{A}\right)^{2}\right]$ (in the approximation $\theta_{\eta} \simeq 0$ );

- $\tilde{\mathcal{A}}^{2}\left[A_{1} \rightarrow 3 \eta^{\prime}\right]=\frac{1}{162} N_{c}\left[\left(\mathcal{Y}_{u}^{A}\right)^{2}+\left(\mathcal{Y}_{d}^{A}\right)^{2}+\left(\mathcal{Y}_{s}^{A}\right)^{2}\right]$ (in the approximation $\theta_{\eta} \simeq 0$ );

- $\tilde{\mathcal{A}}^{2}\left[A_{1} \rightarrow \pi K K\right]=\frac{1}{36} N_{c}\left[4\left(\mathcal{Y}_{u}^{A}\right)^{2}+4\left(\mathcal{Y}_{d}^{A}\right)^{2}+3\left(\mathcal{Y}_{s}^{A}\right)^{2}\right]$

- $\tilde{\mathcal{A}}^{2}\left[A_{1} \rightarrow \eta K K\right]=\frac{1}{12} N_{c}\left[\left(\mathcal{Y}_{s}^{A}\right)^{2}\right]$ (in the approximation $\theta_{\eta} \simeq 0$ );

- $\tilde{\mathcal{A}}^{2}\left[A_{1} \rightarrow \eta^{\prime} K K\right]=\frac{1}{12} N_{c}\left[\left(\mathcal{Y}_{u}^{A}\right)^{2}+\left(\mathcal{Y}_{d}^{A}\right)^{2}+2\left(\mathcal{Y}_{s}^{A}\right)^{2}\right]$ (in the approximation $\theta_{\eta} \simeq 0$ ). 


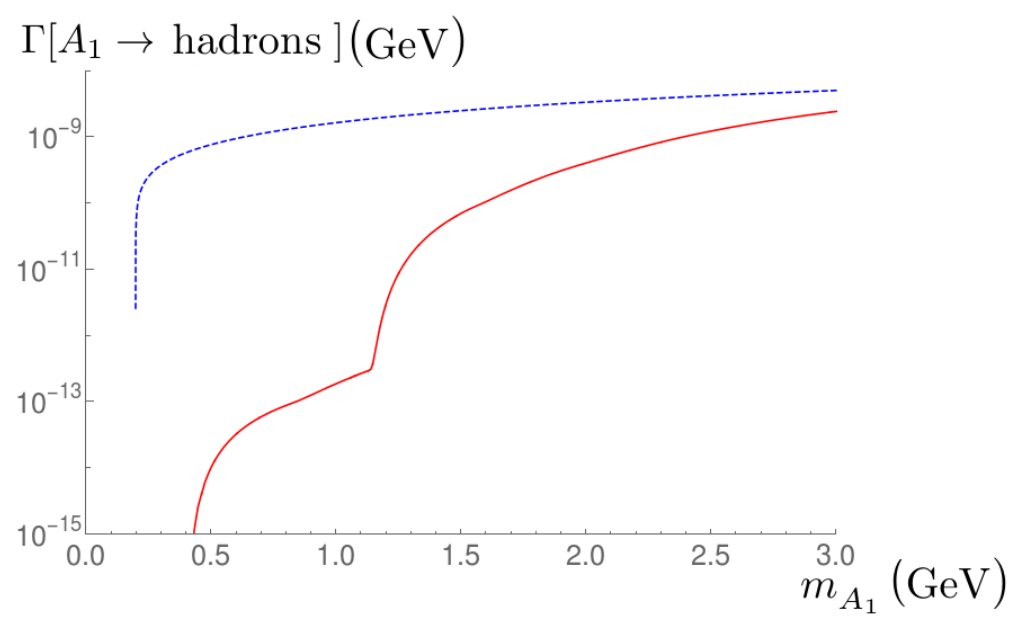

Figure 7. Pure Higgs decays in the spectator approach for $P_{11}=0.03, \tan \beta=10$ : the full red line sums all tri-meson channels. The blue dashed curve corresponds to the two-body decays into quarks obtained for perturbative-quark masses of $m_{u}=2 \mathrm{MeV}, m_{d}=4 \mathrm{MeV}$ and $m_{s}=95 \mathrm{MeV}$.

We may now determine $\mathcal{Y}_{u}^{A}, \mathcal{Y}_{d}^{A}$ and $\mathcal{Y}_{s}^{A}$ from a formal matching of the $A_{1} \rightarrow 3 \pi$ and $A_{1} \rightarrow \pi K K$ amplitudes in the chiral and the spectator approaches, which returns the expressions of eq. (4.4). From this distribution, we can derive the picture of figure 7 , where the sum of the tri-meson decay-widths is shown as a full red curve. It essentially appears as the superposition of two decay widths with respective thresholds $\sim 0.4 \mathrm{GeV}$ (for the light $u$, $d$ quarks) and $\sim 1.1 \mathrm{GeV}$ (for the $s$ quark). Interestingly, this total tri-meson width appears to converge slowly towards the quark-partonic width (blue dashed curve) obtained for perturbative-quark masses of $m_{u}=2 \mathrm{MeV}, m_{d}=4 \mathrm{MeV}$ and $m_{s}=95 \mathrm{MeV}$. A transition to the perturbative quark-regime may thus become relevant around $m_{A_{1}} \gtrsim 3 \mathrm{GeV}$. From this perspective, the impact of mesons for the $A_{1}$ appears as a delayed kinematic opening of the quark decays.

We had temporarily forgotten about the $A_{1}$-meson mixing. This effect is present in eq. (4.1) however, if we evaluate the partonic operators for the meson wave functions. Below, we shall keep the coefficients derived in the chiral limit. It is understood that the mixing effect should disappear slowly as $m_{A_{1}}$ is farther away from the $\eta^{\prime}$ mass. Yet, we have seen in the chiral limit that the sizable $\eta / \eta^{\prime}$ couplings to mesons may extend some influence up to $m_{A_{1}} \sim 1.5 \mathrm{GeV}$. Adding this ingredient to the spectator widths, we arrive at the picture of figure 8. The diphoton (full red line) and the hadronic (green dot-dashed) widths are still under the influence of the $\eta / \eta^{\prime}$ at $m_{A_{1}} \simeq 1 \mathrm{GeV}$ but eventually converge towards a decoupled regime at $m_{A_{1}} \simeq 3 \mathrm{GeV}$. We also observe that the hadronic width eventually becomes competitive with the leptonic one (blue dashed curve) around $m_{A_{1}} \simeq 2.5-3 \mathrm{GeV}$.

Close to $m_{A_{1}} \simeq 3 \mathrm{GeV}$, the charm threshold opens up. The impact on the decays of the CP-odd Higgs is not necessarily large, as the $A_{1} c \bar{c}$ coupling is $\tan \beta$-suppressed. As for the interaction with the lighter quarks - or at the $b \bar{b}$ threshold [56] -, the first effect that can be expected is a mixing of the CP-odd Higgs with the pseudoscalar charmonia $\eta_{c}(n S)$ - in particular $m_{\eta_{c}(1 S)} \simeq 2.98 \mathrm{GeV}$. Then genuine $c \bar{c}$ decays become kinematically allowed when 


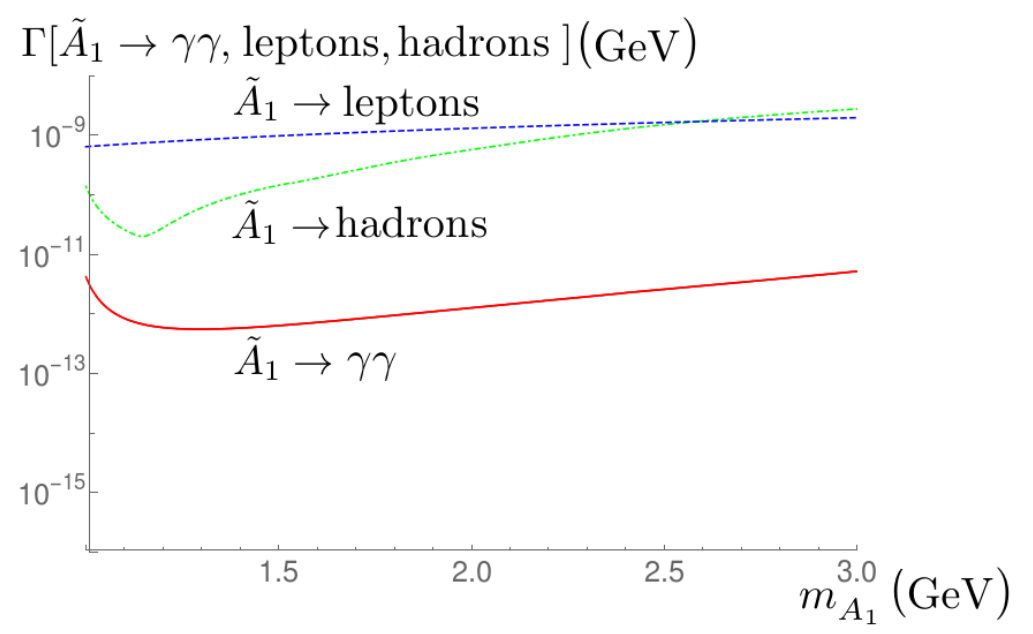

Figure 8. Higgs decays in the spectator approach (including meson-mixing) for $P_{11}=0.03, \tan \beta=$ 10: the green dot-dashed line sums all tri-meson channels. The blue dashed curve corresponds to the leptonic decays and the full red curve to the diphoton width.

$m_{A_{1}} \gtrsim m_{\pi}+2 m_{D} \simeq 3.9 \mathrm{GeV}$. In the meanwhile, however, the $\tau^{+} \tau^{-}$threshold has been reached and the $A_{1} \rightarrow \tau^{+} \tau^{-}$decay, $\tan ^{2} \beta$-enhanced, should dominate the disintegrations of the pseudoscalar Higgs, placing all the hadronic or muonic branching ratios at the percent level. As our focus in this paper is the very-low mass region, we will not detail these effects here.

Before closing this discussion, we display the branching ratios of the light pseudoscalar for $P_{11}=0.03, \tan \beta=10$ in figure 9 , both in the chiral and the spectator approaches. We assume that there is no invisible decay. Expectedly, the leptonic decays (blue dashed curve) dominate over a wide range of mass. However, the diphoton channel can be competitive at low mass, in the vicinity of $m_{A_{1}} \simeq m_{\pi}$ or just below the dimuon threshold. The hadronic decays become sizable at $m_{A_{1}} \simeq 3 \mathrm{GeV}$ or close to the $\eta$ and $\eta^{\prime}$ masses. The total width is shown in the lower plot of figure 9. The general scale is that of the dimuon decay width, but the meson resonances are visible as small spikes. Below the dimuon threshold and with the exception of $m_{A_{1}} \sim m_{\pi}$, the CP-odd Higgs is relatively long-lived. Considering the boost factor of order (at least) $\sim 100$ in LHC searches for $H[125] \rightarrow 2 A_{1}, A_{1}$ would fly centimeters before decaying, leading to displaced vertices. With even larger boost factors (due to e.g. lower $m_{A_{1}}$, sizable longitudinal energy) or smaller $P_{11}$, the pseudoscalar may well escape the detectors, thus appearing as missing energy. Above the dimuon treshold, the CP-odd Higgs is reasonably short-lived and should decay within $\mu \mathrm{m}$, unless $P_{11}$ is extremely small.

It is now time to summarize our achievements in this paper. We have shown how the interactions of a light NMSSM Higgs pseudoscalar with the hadronic sector could be described by a chiral lagrangian. A remarkable effect, which, to our knowledge, has not received much attention before, appears in the form of a mixing of the CP-odd Higgs with the pseudoscalar mesons. This mixing has little effect on the mesons themselves, since they have large hadronic or anomaly-driven decays. On the other hand, its impact on the 


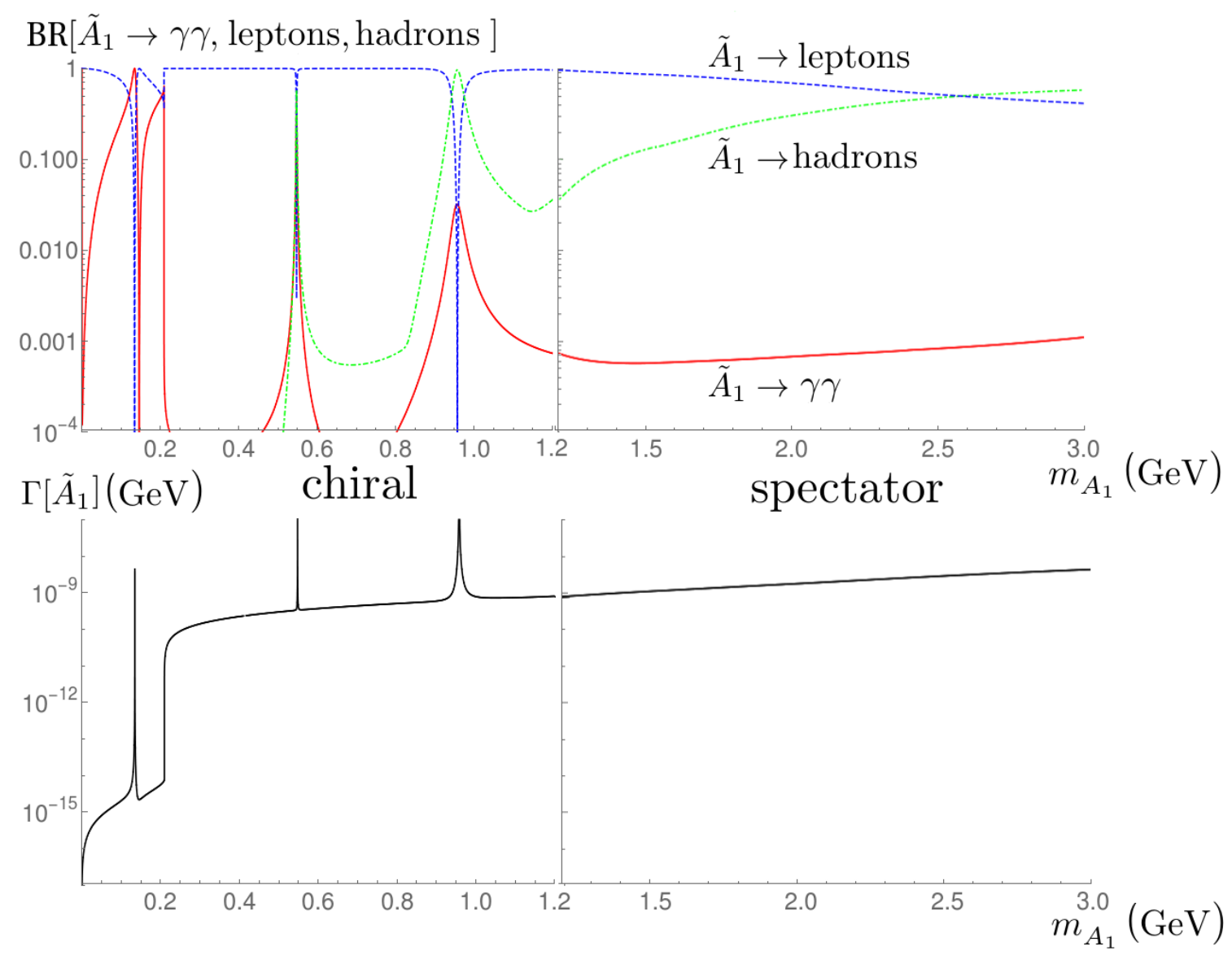

Figure 9. Upper plot: Higgs branching ratios for $P_{11}=0.03, \tan \beta=10$ : hadronic (green dotdashed), leptonic (blue dashed), diphoton (red full). Lower plot: total $A_{1}$ width. The chiral model has been used up to $m_{A_{1}}=1.2 \mathrm{GeV}$. Beyond, we employ the spectator approach.

naturally narrow (singlet-dominated) $A_{1}$ can be very important. In particular, the photonic and hadronic decay widths are sizably affected. However, the leptonic final state remains the dominant decay channel of the Higgs state in most of the considered mass-range. As the mesons do not possess large decays into leptons, it is possible to place limits on the leptonic width that they acquire via mixing. The chiral lagrangian also provides quartic $A_{1^{-}}$ meson interaction terms. These govern the tri-meson decays of the pure-Higgs component. Beyond $m_{A_{1}} \simeq 1 \mathrm{GeV}$, it is possible to extend the description of the Higgs-interactions with the strong-interacting sector using the spectator approach.

Our results can be criticized in many ways. In particular, sizable higher order corrections are known to modify the chiral couplings of the mesons, so that it seems questionable to rely on the tree-level chiral vertices for the Higgs state. A more comprehensive approach, using higher-dimensional chiral operators as well as loop effects, has proved (partially) successful in describing the hadronic decays of the $\eta$ and $\eta^{\prime}$ and the same type of exercise could probably be adapted with profit to the case of the light CP-odd Higgs, increasing the reliability of the computed widths. Yet, such a calculation is far beyond the ambitions of this paper where our scope is limited to a qualitative investigation of the relevant effects. 
Consequently, even though the picture that we propose is somewhat more convincing than a pure partonic, quark-perturbative approach, we should remain aware that large corrections (of order 100\%) could modify the actual magnitude of the hadronic decays.

Deficient they are, our estimates of the pseudoscalar Higgs widths can be applied to constrain the rich phenomenology of this particle. In particular, limits from flavourphysics, quarkonium decays or beam-dump experiment should be considered in this new light. Still, an additional decay channel should be considered in the presence of a very-light Dark Matter candidate. A Fortran implementation of the $A_{1}$-decays in the low-mass region is in preparation at the request of NMSSMTools [93-95].

Finally, we remind that the effects that we have described here in the explicit case of the NMSSM could be easily transposed to other models including a light CP-odd state.

\section{Acknowledgments}

The author thanks U. Ellwanger for comments. This work has been supported by CICYT (grant FPA 2013-40715-P).

Open Access. This article is distributed under the terms of the Creative Commons Attribution License (CC-BY 4.0), which permits any use, distribution and reproduction in any medium, provided the original author(s) and source are credited.

\section{References}

[1] U. Ellwanger, C. Hugonie and A.M. Teixeira, The Next-to-Minimal Supersymmetric Standard Model, Phys. Rept. 496 (2010) 1 [arXiv:0910.1785] [INSPIRE].

[2] P. Bechtle et al., The Light and Heavy Higgs Interpretation of the MSSM, Eur. Phys. J. C 77 (2017) 67 [arXiv: 1608.00638] [INSPIRE].

[3] F. Domingo and G. Weiglein, NMSSM interpretations of the observed Higgs signal, JHEP 04 (2016) 095 [arXiv: 1509.07283] [INSPIRE].

[4] OPAL, DELPHI, LEP Working Group for Higgs boson searches, ALEPH and L3 collaborations, R. Barate et al., Search for the standard model Higgs boson at LEP, Phys. Lett. B 565 (2003) 61 [hep-ex/0306033] [INSPIRE].

[5] ATLAS collaboration, Search for charged Higgs bosons decaying via $H^{ \pm} \rightarrow \tau^{ \pm} \nu$ in fully hadronic final states using pp collision data at $\sqrt{s}=8 \mathrm{TeV}$ with the ATLAS detector, JHEP 03 (2015) 088 [arXiv: 1412.6663] [inSPIRE].

[6] CMS collaboration, Search for a charged Higgs boson in pp collisions at $\sqrt{s}=8$ TeV, JHEP 11 (2015) 018 [arXiv: 1508.07774] [INSPIRE].

[7] B.A. Dobrescu, G.L. Landsberg and K.T. Matchev, Higgs boson decays to CP odd scalars at the Tevatron and beyond, Phys. Rev. D 63 (2001) 075003 [hep-ph/0005308] [INSPIRE].

[8] B.A. Dobrescu and K.T. Matchev, Light axion within the next-to-minimal supersymmetric standard model, JHEP 09 (2000) 031 [hep-ph/0008192] [INSPIRE].

[9] R. Dermisek and J.F. Gunion, The NMSSM Close to the R-symmetry Limit and Naturalness in $h \rightarrow$ aa Decays for $m_{a}<2 m_{b}$, Phys. Rev. D 75 (2007) 075019 [hep-ph/0611142] [INSPIRE]. 
[10] D.E. Morrissey and A. Pierce, Modified Higgs Boson Phenomenology from Gauge or Gaugino Mediation in the NMSSM, Phys. Rev. D 78 (2008) 075029 [arXiv:0807.2259] [inSPIRE].

[11] G. Hiller, B physics signals of the lightest CP odd Higgs in the NMSSM at large tan beta, Phys. Rev. D 70 (2004) 034018 [hep-ph/0404220] [InSPIRE].

[12] F. Domingo and U. Ellwanger, Updated Constraints from B Physics on the MSSM and the NMSSM, JHEP 12 (2007) 090 [arXiv:0710.3714] [InSPIRE].

[13] Z. Heng, R.J. Oakes, W. Wang, Z. Xiong and J.M. Yang, B meson dileptonic decays in the next-to-minimal supersymmetric model with a light CP-odd Higgs boson, Phys. Rev. D 77 (2008) 095012 [arXiv:0801.1169] [INSPIRE].

[14] S. Andreas, O. Lebedev, S. Ramos-Sanchez and A. Ringwald, Constraints on a very light CP-odd Higgs of the NMSSM and other axion-like particles, JHEP 08 (2010) 003 [arXiv: 1005.3978] [INSPIRE].

[15] F. Domingo, Update of the flavour-physics constraints in the NMSSM, Eur. Phys. J. C 76 (2016) 452 [arXiv : 1512.02091] [INSPIRE].

[16] M. Drees and K.-i. Hikasa, Heavy Quark Thresholds in Higgs Physics, Phys. Rev. D 41 (1990) 1547 [INSPIRE].

[17] M.A. Sanchis-Lozano, Searching for new physics in leptonic decays of bottomonium, Mod. Phys. Lett. A 17 (2002) 2265 [hep-ph/0206156] [INSPIRE].

[18] M.A. Sanchis-Lozano, Leptonic universality breaking in $\Upsilon$ decays as a probe of new physics, Int. J. Mod. Phys. A 19 (2004) 2183 [hep-ph/0307313] [inSPIRE].

[19] M.A. Sanchis-Lozano, Searching for new physics in bottomonium decays, PoS (HEP2005) 334 [hep-ph/0510374] [INSPIRE].

[20] B. McElrath, Invisible quarkonium decays as a sensitive probe of dark matter, Phys. Rev. D 72 (2005) 103508 [hep-ph/0506151] [INSPIRE].

[21] M.-A. Sanchis-Lozano, Proposal for testing lepton universality in Upsilon decays at a B-factory running on the Upsilon(3S), J. Phys. Soc. Jap. 76 (2007) 044101 [hep-ph/0610046] [INSPIRE].

[22] R. Dermisek, J.F. Gunion and B. McElrath, Probing NMSSM Scenarios with Minimal Fine-Tuning by Searching for Decays of the $\Upsilon$ to a Light CP-Odd Higgs Boson, Phys. Rev. D 76 (2007) 051105 [hep-ph/0612031] [INSPIRE].

[23] E. Fullana and M.-A. Sanchis-Lozano, Hunting a light CP-odd non-standard Higgs boson through its tauonic decay at a (Super) B factory, Phys. Lett. B 653 (2007) 67 [hep-ph/0702190] [INSPIRE].

[24] R.N. Hodgkinson, $\Upsilon \rightarrow \gamma A_{1}$ in the NMSSM at large $\tan \beta$, Phys. Lett. B 665 (2008) 219 [arXiv: 0802.3197] [INSPIRE].

[25] F. Domingo, U. Ellwanger, E. Fullana, C. Hugonie and M.-A. Sanchis-Lozano, Radiative Upsilon decays and a light pseudoscalar Higgs in the NMSSM, JHEP 01 (2009) 061 [arXiv:0810.4736] [INSPIRE].

[26] D. McKeen, Constraining Light Bosons with Radiative $\Upsilon(1 S)$ Decays, Phys. Rev. D 79 (2009) 015007 [arXiv:0809.4787] [INSPIRE].

[27] F. Domingo, U. Ellwanger and M.-A. Sanchis-Lozano, Bottomoniom spectroscopy with mixing of $\eta_{b}$ states and a light CP-odd Higgs, Phys. Rev. Lett. 103 (2009) 111802 [arXiv:0907.0348] [INSPIRE]. 
[28] R. Dermisek and J.F. Gunion, New constraints on a light CP-odd Higgs boson and related NMSSM Ideal Higgs Scenarios, Phys. Rev. D 81 (2010) 075003 [arXiv:1002.1971] [INSPIRE].

[29] F. Domingo, Updated constraints from radiative $\Upsilon$ decays on a light CP-odd Higgs, JHEP 04 (2011) 016 [arXiv: 1010.4701] [INSPIRE].

[30] M.M. Almarashi and S. Moretti, Scope of Higgs production in association with a bottom quark pair in probing the Higgs sector of the NMSSM at the LHC, arXiv:1205.1683 [INSPIRE].

[31] J. Rathsman and T. Rossler, Closing the Window on Light Charged Higgs Bosons in the NMSSM, Adv. High Energy Phys. 2012 (2012) 853706 [arXiv:1206.1470] [INSPIRE].

[32] D.G. Cerdeno, P. Ghosh and C.B. Park, Probing the two light Higgs scenario in the NMSSM with a low-mass pseudoscalar, JHEP 06 (2013) 031 [arXiv:1301.1325] [INSPIRE].

[33] N.-E. Bomark, S. Moretti, S. Munir and L. Roszkowski, A light NMSSM pseudoscalar Higgs boson at the LHC redux, JHEP 02 (2015) 044 [arXiv: 1409. 8393] [INSPIRE].

[34] N.-E. Bomark, S. Moretti and L. Roszkowski, Detection prospects of light NMSSM Higgs pseudoscalar via cascades of heavier scalars from vector boson fusion and Higgs-strahlung, $J$. Phys. G 43 (2016) 105003 [arXiv: 1503. 04228] [inSPIRE].

[35] E. Conte, B. Fuks, J. Guo, J. Li and A.G. Williams, Investigating light NMSSM pseudoscalar states with boosted ditau tagging, JHEP 05 (2016) 100 [arXiv: 1604.05394] [INSPIRE].

[36] ATLAS collaboration, Observation of a new particle in the search for the Standard Model Higgs boson with the ATLAS detector at the LHC, Phys. Lett. B 716 (2012) 1 [arXiv: 1207.7214] [INSPIRE].

[37] CMS collaboration, Observation of a new boson at a mass of $125 \mathrm{GeV}$ with the CMS experiment at the LHC, Phys. Lett. B 716 (2012) 30 [arXiv:1207.7235] [INSPIRE].

[38] J. Cao, F. Ding, C. Han, J.M. Yang and J. Zhu, A light Higgs scalar in the NMSSM confronted with the latest LHC Higgs data, JHEP 11 (2013) 018 [arXiv:1309.4939] [INSPIRE].

[39] ATLAS collaboration, Search for Higgs bosons decaying to aa in the $\mu \mu \tau \tau$ final state in $p p$ collisions at $\sqrt{s}=8 \mathrm{TeV}$ with the ATLAS experiment, Phys. Rev. D 92 (2015) 052002 [arXiv: 1505.01609] [INSPIRE].

[40] CMS collaboration, A search for pair production of new light bosons decaying into muons, Phys. Lett. B 752 (2016) 146 [arXiv: 1506.00424] [INSPIRE].

[41] CMS collaboration, Search for a very light NMSSM Higgs boson produced in decays of the $125 \mathrm{GeV}$ scalar boson and decaying into $\tau$ leptons in pp collisions at $\sqrt{s}=8 \mathrm{TeV}$, JHEP 01 (2016) 079 [arXiv: 1510.06534] [INSPIRE].

[42] D.G. Cerdeno, C. Hugonie, D.E. Lopez-Fogliani, C. Muñoz and A.M. Teixeira, Theoretical predictions for the direct detection of neutralino dark matter in the NMSSM, JHEP 12 (2004) 048 [hep-ph/0408102] [INSPIRE].

[43] G. Bélanger, F. Boudjema, C. Hugonie, A. Pukhov and A. Semenov, Relic density of dark matter in the NMSSM, JCAP 09 (2005) 001 [hep-ph/0505142] [INSPIRE].

[44] D.G. Cerdeno, E. Gabrielli, D.E. Lopez-Fogliani, C. Muñoz and A.M. Teixeira, Phenomenological viability of neutralino dark matter in the NMSSM, JCAP 06 (2007) 008 [hep-ph/0701271] [INSPIRE].

[45] C. Hugonie, G. Bélanger and A. Pukhov, Dark matter in the constrained NMSSM, JCAP 11 (2007) 009 [arXiv:0707.0628] [INSPIRE]. 
[46] D. Albornoz Vasquez, G. Bélanger, C. Boehm, A. Pukhov and J. Silk, Can neutralinos in the MSSM and NMSSM scenarios still be light?, Phys. Rev. D 82 (2010) 115027 [arXiv: 1009.4380] [INSPIRE].

[47] J.-J. Cao et al., Light dark matter in NMSSM and implication on Higgs phenomenology, Phys. Lett. B 703 (2011) 292 [arXiv:1104.1754] [INSPIRE].

[48] D. Albornoz Vasquez, G. Bélanger, C. Boehm, J. Da Silva, P. Richardson and C. Wymant, The $125 \mathrm{GeV}$ Higgs in the NMSSM in light of LHC results and astrophysics constraints, Phys. Rev. D 86 (2012) 035023 [arXiv: 1203.3446] [inSPIRE].

[49] T. Han, Z. Liu and S. Su, Light Neutralino Dark Matter: Direct/Indirect Detection and Collider Searches, JHEP 08 (2014) 093 [arXiv:1406.1181] [INSPIRE].

[50] U. Ellwanger and C. Hugonie, The semi-constrained NMSSM satisfying bounds from the LHC, LUX and Planck, JHEP 08 (2014) 046 [arXiv: 1405.6647] [INSPIRE].

[51] C. Han, D. Kim, S. Munir and M. Park, O(1) GeV dark matter in SUSY and a very light pseudoscalar at the LHC, JHEP 07 (2015) 002 [arXiv: 1504.05085] [INSPIRE].

[52] D.G. Cerdeno, M. Peiro and S. Robles, Enhanced lines and box-shaped features in the gamma-ray spectrum from annihilating dark matter in the NMSSM, JCAP 04 (2016) 011 [arXiv: 1507.08974] [INSPIRE].

[53] U. Ellwanger and S. Moretti, Possible Explanation of the Electron Positron Anomaly at $17 \mathrm{MeV}$ in ${ }^{8}$ Be Transitions Through a Light Pseudoscalar, JHEP 11 (2016) 039 [arXiv: 1609.01669] [INSPIRE].

[54] A.J. Krasznahorkay et al., Observation of Anomalous Internal Pair Creation in ${ }^{8} B e: A$ Possible Indication of a Light, Neutral Boson, Phys. Rev. Lett. 116 (2016) 042501 [arXiv: 1504.01527] [INSPIRE].

[55] M.J. Dolan, F. Kahlhoefer, C. McCabe and K. Schmidt-Hoberg, A taste of dark matter: Flavour constraints on pseudoscalar mediators, JHEP 03 (2015) 171 [Erratum ibid. 07 (2015) 103] [arXiv: 1412.5174] [INSPIRE].

[56] F. Domingo and U. Ellwanger, Reduced branching ratio for $H \rightarrow A A \rightarrow 4 \tau$ from $A-\eta_{b}$ mixing, JHEP 06 (2011) 067 [arXiv: 1105.1722] [INSPIRE].

[57] U. Ellwanger and C. Hugonie, A $750 \mathrm{GeV}$ Diphoton Signal from a Very Light Pseudoscalar in the NMSSM, JHEP 05 (2016) 114 [arXiv: 1602.03344] [INSPIRE].

[58] F. Domingo, S. Heinemeyer, J.S. Kim and K. Rolbiecki, The NMSSM lives: with the $750 \mathrm{GeV}$ diphoton excess, Eur. Phys. J. C 76 (2016) 249 [arXiv: 1602.07691] [INSPIRE].

[59] C. Rosenzweig, J. Schechter and C.G. Trahern, Is the Effective Lagrangian for QCD a o-model?, Phys. Rev. D 21 (1980) 3388 [INSPIRE].

[60] P. Di Vecchia and G. Veneziano, Chiral Dynamics in the Large-N Limit, Nucl. Phys. B 171 (1980) 253 [INSPIRE].

[61] K. Kawarabayashi and N. Ohta, The Problem of $\eta$ in the Large-N Limit: Effective Lagrangian Approach, Nucl. Phys. B 175 (1980) 477 [INSPIRE].

[62] K. Kawarabayashi and N. Ohta, On the Partial Conservation of the U(1) Current, Prog. Theor. Phys. 66 (1981) 1789 [inSPIRE].

[63] J. Gasser and H. Leutwyler, Chiral Perturbation Theory: Expansions in the Mass of the Strange Quark, Nucl. Phys. B 250 (1985) 465 [INSPIRE].

[64] J.R. Ellis, M.K. Gaillard and D.V. Nanopoulos, A Phenomenological Profile of the Higgs Boson, Nucl. Phys. B 106 (1976) 292 [InSPIRE]. 
[65] M.A. Shifman, A.I. Vainshtein, M.B. Voloshin and V.I. Zakharov, Low-Energy Theorems for Higgs Boson Couplings to Photons, Sov. J. Nucl. Phys. 30 (1979) 711 [Yad. Fiz. 30 (1979) 1368] [INSPIRE].

[66] A.I. Vainshtein, V.I. Zakharov and M.A. Shifman, Higgs Particles, Sov. Phys. Usp. 23 (1980) 429 [INSPIRE].

[67] M.B. Voloshin, Once Again About the Role of Gluonic Mechanism in Interaction of Light Higgs Boson with Hadrons, Sov. J. Nucl. Phys. 44 (1986) 478 [inSPIRE].

[68] M.B. Voloshin, Trace anomaly in QCD and processes with a light Higgs boson, Sov. J. Nucl. Phys. 45 (1987) 122 [Yad. Fiz. 45 (1987) 190] [INSPIRE].

[69] R. Ruskov, The Gluonic Mechanism in the Interaction of a Light Higgs Boson With Light Hadrons in the Decays $K^{+} \rightarrow \pi^{+} H, \eta^{\prime} \rightarrow \eta H$, Phys. Lett. B 187 (1987) 165 [INSPIRE].

[70] B. Grinstein, L.J. Hall and L. Randall, Do B meson decays exclude a light Higgs?, Phys. Lett. B 211 (1988) 363 [INSPIRE].

[71] R.S. Chivukula and A.V. Manohar, Limits on a light Higgs boson, Phys. Lett. B 207 (1988) 86 [Erratum ibid. B 217 (1989) 568] [INSPIRE].

[72] R.S. Chivukula, A.G. Cohen, H. Georgi, B. Grinstein and A.V. Manohar, Higgs decay into Goldstone bosons, Annals Phys. 192 (1989) 93 [InSPIRE].

[73] H. Leutwyler and M.A. Shifman, Goldstone bosons generate peculiar conformal anomalies, Phys. Lett. B 221 (1989) 384 [INSPIRE].

[74] S. Dawson, Higgs Boson Production in Semileptonic K and $\pi$ Decays, Phys. Lett. B 222 (1989) 143 [INSPIRE].

[75] H. Leutwyler and M.A. Shifman, Light Higgs Particle in Decays of $K$ and $\eta$ Mesons, Nucl. Phys. B 343 (1990) 369 [INSPIRE].

[76] A. Pich, J. Prades and P. Yepes, Bounds on a light scalar in two Higgs doublet models, Nucl. Phys. B 388 (1992) 31 [INSPIRE].

[77] B. Grzadkowski and J. Pawełczyk, Light elementary pseudoscalars within the framework of effective Lagrangians, Phys. Lett. B 300 (1993) 387 [INSPIRE].

[78] Q. Chang and Y.-D. Yang, Rare decay $\pi^{0} \rightarrow e^{+} e^{-}$as a sensitive probe of light CP-odd Higgs in NMSSM, Phys. Lett. B 676 (2009) 88 [arXiv:0808.2933] [INSPIRE].

[79] G. Grilli di Cortona, E. Hardy, J. Pardo Vega and G. Villadoro, The QCD axion, precisely, JHEP 01 (2016) 034 [arXiv: 1511.02867] [INSPIRE].

[80] J. Wess and B. Zumino, Consequences of anomalous Ward identities, Phys. Lett. 37B (1971) 95 [INSPIRE].

[81] E. Witten, Global Aspects of Current Algebra, Nucl. Phys. B 223 (1983) 422 [InSPIRE].

[82] F. Ambrosino et al., A global fit to determine the pseudoscalar mixing angle and the gluonium content of the eta-prime meson, JHEP 07 (2009) 105 [arXiv:0906.3819] [INSPIRE].

[83] G. Ricciardi, Semileptonic D decays and $\eta-\eta^{\prime}$ mixing, Phys. Rev. D 86 (2012) 117505 [arXiv: 1209.3386] [INSPIRE].

[84] T.N. Pham, $\eta-\eta^{\prime}$ mixing, Phys. Rev. D 92 (2015) 054021 [arXiv:1504.05414] [InSPIRE].

[85] Particle Data Group collaboration, C. Patrignani et al., Review of Particle Physics, Chin. Phys. C 40 (2016) 100001 [INSPIRE].

[86] J.-M. Gerard, C. Smith and S. Trine, Radiative kaon decays and the penguin contribution to the $\Delta I=1 / 2$ rule, Nucl. Phys. B 730 (2005) 1 [hep-ph/0508189] [INSPIRE]. 
[87] KTeV collaboration, E. Abouzaid et al., Measurement of the Rare Decay $\pi^{0} \rightarrow e^{+} e^{-}$, Phys. Rev. D 75 (2007) 012004 [hep-ex/0610072] [INSPIRE].

[88] A.E. Dorokhov and M.A. Ivanov, Rare decay $\pi^{0} \rightarrow e^{+} e^{-}$: Theory confronts KTeV data, Phys. Rev. D 75 (2007) 114007 [arXiv:0704.3498] [InSPIRE].

[89] J.A. Cronin, Phenomenological model of strong and weak interactions in chiral $\mathrm{U}(3) \times \mathrm{U}(3)$, Phys. Rev. 161 (1967) 1483 [INSPIRE].

[90] H. Osborn and D.J. Wallace, $\eta-x$ mixing, eta $\rightarrow 3 \pi$ and chiral lagrangians, Nucl. Phys. B 20 (1970) 23 [INSPIRE].

[91] B.R. Holstein, Allowed eta decay modes and chiral symmetry, Phys. Scripta T 99 (2002) 55 [hep-ph/0112150] [INSPIRE].

[92] J.F. Gunion, H.E. Haber, G.L. Kane and S. Dawson, The Higgs Hunter's Guide, Front. Phys. 80 (2000) 1 [INSPIRE].

[93] U. Ellwanger, J.F. Gunion and C. Hugonie, NMHDECAY: A Fortran code for the Higgs masses, couplings and decay widths in the NMSSM, JHEP 02 (2005) 066 [hep-ph/0406215] [INSPIRE].

[94] U. Ellwanger and C. Hugonie, NMHDECAY 2.0: An Updated program for sparticle masses, Higgs masses, couplings and decay widths in the NMSSM, Comput. Phys. Commun. 175 (2006) 290 [hep-ph/0508022] [inSPIRE].

[95] http://www.th.u-psud.fr/NMHDECAY/nmssmtools.html. 